\title{
Human four-and-a-half LIM family members suppress tumor cell growth through a TGF- $\beta$-like signaling pathway
}

\author{
Lihua Ding, ${ }^{1}$ Zhaoyun Wang, ${ }^{1}$ Jinghua Yan, ${ }^{1}$ Xiao Yang, ${ }^{1}$ Aijun Liu, ${ }^{2}$ Weiyi Qiu, ${ }^{1}$ Jianhua Zhu, ${ }^{3}$ \\ Juqiang Han, ${ }^{4}$ Hao Zhang, ${ }^{1}$ Jing Lin, ${ }^{5}$ Long Cheng, ${ }^{1}$ Xi Qin, ${ }^{1}$ Chang Niu, ${ }^{1}$ Bin Yuan, ${ }^{1}$ \\ Xiaohui Wang, ${ }^{1}$ Cui Zhu, ${ }^{1}$ Yan Zhou, ${ }^{1}$ Jiezhi Li, ${ }^{1}$ Haifeng Song, ${ }^{6}$ Cuifen Huang, ${ }^{1}$ and Qinong Ye ${ }^{1}$ \\ 'Beijing Institute of Biotechnology, Beijing, People's Republic of China. ${ }^{2}$ Department of Pathology and \\ ${ }^{3}$ Department of Oncology, First Affiliated Hospital, China PLA General Hospital, Beijing, People's Republic of China. \\ ${ }^{4}$ Institute of Hepatology, Beijing Military General Hospital, Beijing, People's Republic of China. ${ }^{5}$ Department of Clinical Laboratory, First Affiliated Hospital, \\ China PLA General Hospital, Beijing, People's Republic of China. ${ }^{6}$ Beijing Institute of Radiation Medicine, Beijing, People's Republic of China.
}

The four-and-a-half LIM (FHL) proteins belong to a family of LIM-only proteins that regulate cell proliferation, differentiation, and apoptosis. The exact functions of each FHL protein in cancer development and progression remain unknown. Here we report that FHL1, FHL2, and FHL3 physically and functionally interact with Smad2, Smad3, and Smad4, important regulators of cancer development and progression, in a TGF- $\beta$ independent manner. Casein kinase $1 \delta$, but not the TGF- $\beta$ receptor, was required for the FHL-mediated TGF- $\beta$ like responses, including increased phosphorylation of Smad2/3, interaction of Smad2/3 and Smad4, nuclear accumulation of Smad proteins, activation of the tumor suppressor gene $p 21$, and repression of the oncogene $c$-myc. FHL1-3 inhibited anchorage-dependent and -independent growth of a human hepatoma cell line in vitro and tumor formation in nude mice. Further analysis of clinical samples revealed that FHL proteins are often downregulated in hepatocellular carcinomas and that this correlates with decreased TGF- $\beta$-like responses. By establishing a link between FHL proteins and Smad proteins, this study identifies what we believe to be a novel TGF- $\beta$-like signaling pathway and indicates that FHL proteins may be useful molecular targets for cancer therapy.

\section{Introduction}

The four-and-a-half LIM (FHL) proteins are characterized by 4 complete LIM domains preceded by an N-terminal half LIM domain (1). LIM domains are cysteine-rich zinc finger motifs involved in a wide range of protein-protein interactions. Amino acid sequence comparisons reveal that FHL proteins are more than $40 \%$ identical. Through interaction with cellular proteins, FHL proteins regulate cellular processes, including proliferation, differentiation, apoptosis, adhesion, migration, transcription, and signal transduction (2-10). Recently, FHL proteins have been shown to play roles in carcinogenesis. FHL1 suppression is required for Src to promote mouse cancer cell growth and migration (11). Although the clinical sample number is unknown, FHL1 expression was downregulated in several types of human tumors, including astrocytoma, breast carcinoma, renal carcinoma, hepatocarcinoma, pulmonary adenocarcinoma, prostatic carcinoma, and melanoma (11). The role of FHL2 in tumorigenesis is complex, as human FHL2 is downregulated in malignant rhabdomyosarcoma but upregulated in ovarian and gastrointestinal cancers (1). Ectopic expression of FHL2 induces apoptosis in the human embryonic rhabdomyosarcoma cell line RD, NIH 3T3 mouse fibroblasts, and COS-1 African green monkey kidney cells (12). However, stable transfection of gastric and colon

Conflict of interest: The authors have declared that no conflict of interest exists. Nonstandard abbreviations used: CK1 $\delta$, casein kinase $1 \delta$; CLIM-1, LIM homeobox protein cofactor; FHL, four-and-a-half LIM; GST, glutathione-S-transferase; HCC hepatocellular carcinoma; His-, His-tagged; lac, lactose repressor; MH1, Mad homology 1; PAI-1, plasminogen activator inhibitor-1; RBPMS, RNA-binding protein with multiple splicing; T $\beta$ R-I, TGF- $\beta$ type I receptor.

Citation for this article: J. Clin. Invest. 119:349-361 (2009). doi:10.1172/JCI35930. cancer cell lines with antisense FHL2 inhibits anchorage-dependent cell growth, and stable transfectants display significantly reduced anchorage-independent growth. Additionally, FHL2 suppression in colon cancer cells inhibits tumorigenesis in nude mice (13). Although FHL1 and FHL2 may play roles in cancer development and progression, the detailed mechanisms of their functions and the roles of other FHL proteins in cancer remain unknown.

Smad proteins, including Smad2, Smad3, and Smad4, mediate TGF- $\beta$ signaling and regulate cell proliferation, differentiation, migration, apoptosis, and development (14-16). Smads have 2 highly conserved domains at the $\mathrm{N}$ terminus and the $\mathrm{C}$ terminus, referred to as Mad homology 1 (MH1) and MH2 domains, respectively. Both domains are separated by a variable, flexible linker domain, rich in proline residues. The MH1 domain is responsible for DNA binding, whereas the MH2 domain has trans-activation activity. TGF- $\beta$ binds to a heteromeric receptor complex, consisting of type I and type II transmembrane receptor serine/threonine kinases. Upon ligand binding, the type II receptor kinase phosphorylates and activates the type I receptor kinase, which subsequently phosphorylates the cytoplasmic Smad2 and Smad3 proteins at the C-terminal SSXS regions. Receptor-activated Smad2 and Smad3 undergo a conformational change that allows heteromerization with Smad4. The activated complex subsequently translocates to the nucleus, in which the Smad proteins regulate the expression of target genes, including plasminogen activator inhibitor-1 (PAI-1), the cyclin-dependent kinase inhibitors $p 15$ and $p 21$, and the oncogene $c-m y c$, through their interaction with a variety of transcription factors, coactivators, and corepressors (17-19). Alterations in Smad2 and Smad4 genes have been identi- 
fied in many cancer types, including pancreatic, breast, ovarian, colorectal, lung, gastrointestinal, and liver cancer. However, how these genes suppress tumor growth is not fully understood.

We report that FHL1, FHL2, and FHL3 physically and functionally interact with Smad 2 , Smad3, and Smad 4 in TGF- $\beta$ - and TGF- $\beta$ receptor-independent manners. Casein kinase $1 \delta$ (CK1 $\delta$ ), but not the TGF- $\beta$ receptor, is required for FHL-mediated TGF- $\beta$-like responses (20). FHL1-3 inhibit hepatocellular carcinoma (HCC) cell growth, both in vitro and in nude mice. Furthermore, FHL protein expression is downregulated in patients with HCC and correlates with FHL-mediated TGF- $\beta$-like responses.

\section{Results}

Interaction of FHL proteins with Smad proteins in vitro and in vivo. FHL2 was shown to bind Smad4 in the yeast 2-hybrid system. Since FHL2 shares similarity with FHL1 and FHL3 and Smad2, Smad3, and Smad4 share conserved MH1 and MH2 regions, the possibility that FHL proteins interact with Smad proteins was investigated using glutathione-S-transferase (GST) pull-down assays. As expected, the purified His-tagged Smad2-4 (His-Smad2-4) proteins bound to GST-FHL1-3, with comparable binding affinity, but not to GST or GST fused to the LIM homeobox protein cofactor CLIM-1, indicating that direct interaction between Smads and FHL proteins is specific (Figure 1A).

To investigate FHL and Smad protein interaction in mammalian cells, coimmunoprecipitation assays were performed with human embryonic kidney 293T cells. FLAG-tagged FHL1, FHL2, and FHL3 coimmunoprecipitated lactose repressor-Smad2 (lac-Smad2), lacSmad3, and lac-Smad4 in a TGF- $\beta$-independent manner (Figure 1, B-D). As a control, FLAG-CLIM-1 failed to coimmunoprecipitate lac-Smad4 (Figure 1E). The predicted molecular weights of FLAGtagged FHL1, FHL2, and FHL3 were $33 \mathrm{kDa}, 33 \mathrm{kDa}$, and $32 \mathrm{kDa}$, respectively. However, immunoblotting with anti-FLAG antibody revealed that FLAG-FHL3 migrated the slowest of the 3 tagged proteins, possibly due to different posttranslational modifications of FHL proteins (Figure 1, B-D). The physiological interaction of FHL and Smad proteins was confirmed by coimmunoprecipitation assays with human hepatoma HepG2 cells. Endogenous FHL1, FHL2, or FHL3 proteins coprecipitated with Smad2/3 or Smad4, from both cytoplasmic and nuclear fractions, in a TGF- $\beta$-independent manner (Figure $1 \mathrm{~F}$ and data not shown).

FHL mediates TGF- $\beta$-responsive transcription and the promoter occupancy of Smad proteins. As FHL1, FHL2, and FHL3 have similar functions, in some cases, we present data for FHL1 only. To investigate whether the FHL1-Smad interaction affects TGF- $\beta$-responsive gene transcription, TGF- $\beta$-responsive HepG 2 cells were cotransfected with the reporters $\mathrm{p} 3 \mathrm{TP}$-Lux (synthetic TGF- $\beta$-responsive reporter), wwp-Luc (p21 promoter-containing reporter), or $\mathrm{PHX}-\mathrm{Luc}$ (c-myc promotercontaining reporter) and FLAG-tagged FHL1 or FHL1 siRNA. With and without TGF- $\beta$, FHL1 overexpression enhanced P3TP-Lux and wwp-Luc reporter activity and decreased $\mathrm{pHX}$-Luc reporter activity (Figure 2, A and B, and data not shown), whereas siRNA knockdown of endogenous FHL1 decreased p3TP-Lux and wwp-Luc reporter activity and enhanced $\mathrm{pHX}$-Luc reporter activity (Figure $2 \mathrm{C}$ ). Therefore, FHL1 enhances TGF- $\beta /$ Smad-mediated signaling. As controls, CLIM-1 showed no effect (Figure 2, A and B), and FHL1 did not increase the activity of the estrogen-responsive reporter, ERE-Luc (data not shown), which cross-talks with TGF- $\beta /$ Smad-mediated signaling (21). Interestingly, FHL1, FHL2, and FHL3 have additive effects on wwp-Luc reporter activity in HepG2 cells (Supplemental
Figure 1, A and B; supplemental material available online with this article; doi:10.1172/JCI35930DS1). Similar results were observed in the hepatoma cell lines, Hep3B and SMMC7721, and the embryonic kidney cell line, 293T (data not shown). Moreover, using a lac repressor-lac operator recognition system (22), FHL1 directly enhanced the transcriptional activity of lac-Smad2, lac-Smad3, or lac-Smad4 in a TGF- $\beta$-independent manner (Figure 2D).

To assess the role of FHL1 as a coactivator of Smad-mediated transcription, ChIP experiments were performed for the PAI-1, $p 21$, and $c-m y c$ promoters. Like Smad2/3 or Smad4, FHL1 was recruited to the PAI-1, 221 , and $c-m y c$ promoters, but not to a region approximately $2-\mathrm{kb}$ upstream of the PAI-1, $p 21$, or $c-m y c$ promoters, albeit in a TGF- $\beta$-independent manner (Supplemental Figure 1C). Unlike FLAG-tagged FHL1, FLAG-tagged CLIM-1 did not associate with the PAI-1, p21, or c-myc promoter sequences (Supplemental Figure 1D). Importantly, FHL1 overexpression increased the Smad2/3 or Smad4 promoter occupancy (Figure 2E), whereas siRNA knockdown of endogenous FHL1 reduced Smad protein binding to the promoters (Supplemental Figure 1E).

FHL1 and Smad4 interaction is required for activation of TGF- $\beta$-responsive transcription. To determine whether the interaction of FHL1 and Smad4, a central mediator in TGF- $\beta$ signaling, regulates TGF- $\beta$ responsive transcription, we used deletion analysis to map the interaction domains of Smad4 in coimmunoprecipitation assays. The Smad4 $260-552$ fragment containing the MH2 domain bound specifically to FHL1. In contrast, the Smad4 $4_{1-150}$ fragment containing the MH1 domain and the Smad4 ${ }_{130-325}$ fragment containing the linker did not bind FHL1 (Supplemental Figure 2A), suggesting that amino acids $260-325$ and $514-552$ of Smad4 are not required for interaction with FHL1. Further deletion analysis showed that both Smad4 $4_{325-412}$ and Smad4 $412-514$ sufficiently bound FHL1. A protein sequence homology search (www.ncbi.nlm.nih.gov/blast; data not shown) indicated that $\operatorname{Smad}_{325-412}$ and Smad4 $412-514$ share $55.7 \%$ and $13.6 \%$ identity, respectively, with the corresponding Smad 2 and Smad3 regions. Next we determined which FHL1 protein region mediates interaction with Smad4. Deletion of any LIM domain from either the $\mathrm{N}$ - or C-terminal reduced or abolished FHL1 association with Smad4, indicating that both the $\mathrm{N}$ - and C-terminal LIM domains of FHL1 are required for maximal interaction with Smad4 (Supplemental Figure 2B). Importantly, the 2 FHL1 mutants, FHL1 $1_{56-280}$ and FHL1 $1_{1-169}$, which failed to bind Smad4, completely abolished p3TP-Lux reporter activity (Supplemental Figure 2C). Interestingly, 2 pancreatic tumor-derived Smad4 mutations in the MH2 domain, V370D and D493H, which impair both homo- and hetero-oligomerization of $\operatorname{Smad} 4(23,24)$, greatly reduced FHL1-Smad4 interaction (Supplemental Figure 2D) and nearly abolished the ability of FHL1 to increase Smad4 transcriptional activity (Supplemental Figure 2E).

To further investigate whether Smad4 is required for FHL1mediated p3TP-Lux reporter activity, human breast cancer MDAMB-468 cells, which lack endogenous Smad4 (25), were used in the p3TP-Lux assay. Cotransfection of FHL1 and the p3TP-Lux reporter into MDA-MB-468 cells did not increase p3TP-Lux reporter transcription, whereas cotransfection of these genes with Smad4 activated p3TP-Lux (Supplemental Figure 2F). These data strongly suggest that FHL1 acts through Smad4 to increase TGF- $\beta$ responsive transcription.

TGF- $\beta$ - and TGF- $\beta$ receptor-independent regulation of TGF- $\beta$-responsive transcription and Smad2/3 phosphorylation by FHL1. Since FHL1 regulates TGF- $\beta$-responsive transcription in the presence or absence of 
A

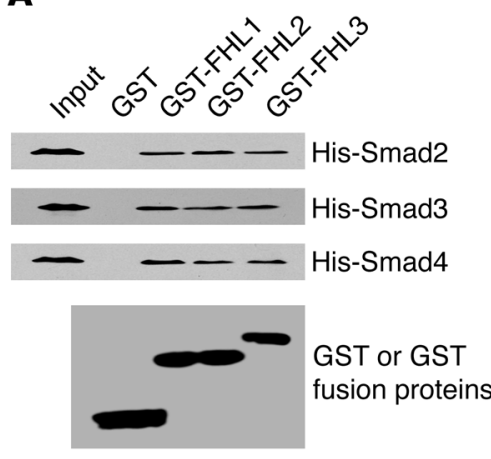

B $\quad$ FLAG- $-\frac{\text { FHL1 }}{\text { TGF- } \beta} \frac{\text { FHL2 }}{-++} \frac{\text { FHL3 }}{-+}$

Lac-Smad2 $\quad-\infty-\infty-\cdots$ IP: $\alpha$-FLAG

Lac-Smad2 $-\infty-\infty-\infty$ Input

$-\infty \quad$ IP: $\alpha$-FLAG

$\longrightarrow$ Input

Input
IB: $\alpha$-FLAG

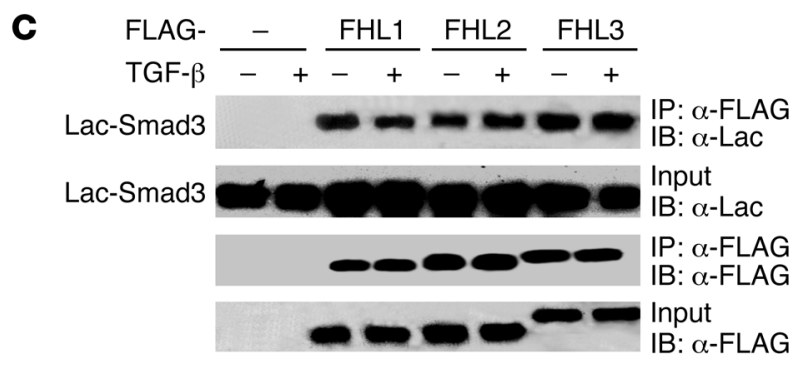

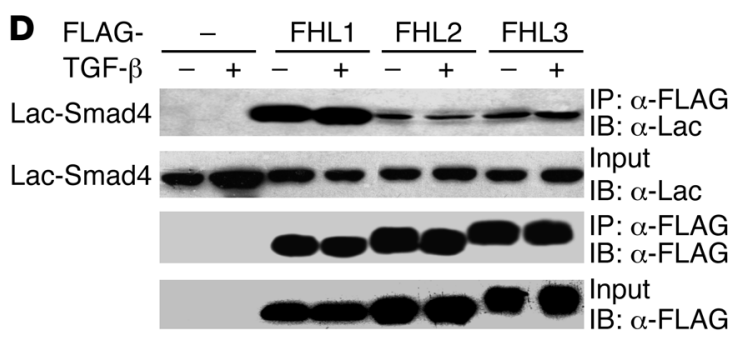

$\mathbf{E}$

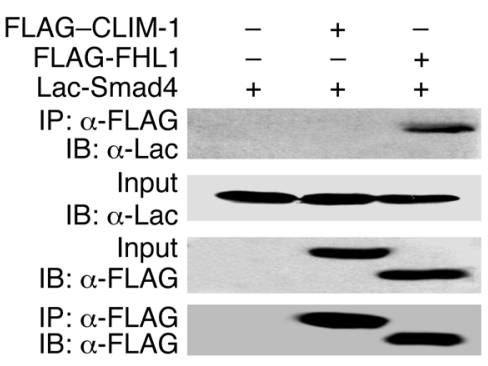

$\mathbf{F}$

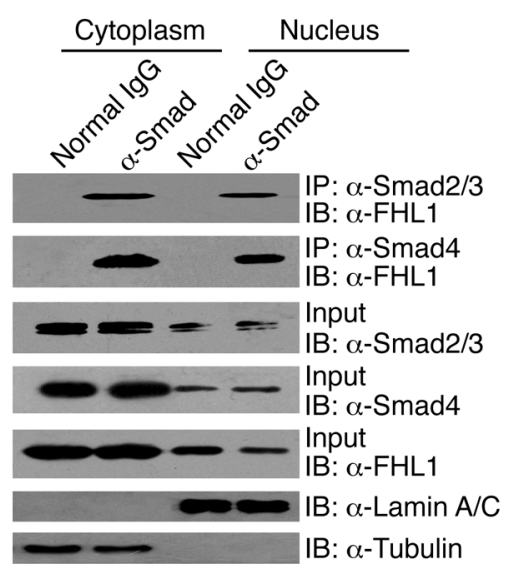

Figure 1

FHL proteins interact with Smad proteins in vitro and in vivo. (A) For the GST pull-down assay, GST or GST fusion proteins were incubated with purified His-Smad proteins. Bound proteins were subjected to SDS-PAGE and Western blot with anti-His antibody. CLIM-1 served as a negative control. (B-E) For the coimmunoprecipitation assay, FLAG-tagged FHL1, FHL2, FHL3, or CLIM-1, and lac-tagged Smad2 (B), Smad3 (C), or Smad4 (D and E) were cotransfected into 293T cells. Cell lysates were immunoprecipitated by anti-FLAG antibody, and precipitates were immunoblotted with anti-lac antibody. CLIM-1 served as a negative control. (F) HepG2 cells were fractionated, and proteins were immunoprecipitated from cytoplasmic and nuclear fractions, with the indicated antibodies or preimmune control serum (IgG). Precipitates were analyzed by immunoblot using the indicated antibodies. Lamin A/C and $\alpha$-tubulin were used as the nuclear and cytoplasmic marker, respectively. The molecular weights were as follows: His-Smad2, $57 \mathrm{kDa}$; His-Smad3, 52 kDa; His-Smad4, $63 \mathrm{kDa}$; GST, 29 kDa; GST-FHL1, 58 kDa; GST-CLIM-1, 71 kDa; FLAG-FHL1, 33 kDa; FLAG-FHL2, 33 kDa; FLAG-FHL3, 36 kDa; FLAG-CLIM-1, 46 kDa; lac-Smad2, 96 kDa; lac-Smad3, 91 kDa; lac-Smad4, 102 kDa; FHL1, 32 kDa; Smad2/Smad3, 56 kDa/51 kDa; Smad4, 62 kDa.

TGF- $\beta$, we sought to determine whether FHL modulation of TGF- $\beta$ responsive transcription is TGF- $\beta$ - and TGF- $\beta$ receptor-independent. As shown in Figure 3A, TGF- $\beta$-neutralizing antibody did not affect FHL1 modulation of p3TP-Lux reporter activity, although it completely abolished reporter activity regulated by RNA-binding protein with multiple splicing (RBPMS), a protein that regulates this reporter activity in a TGF- $\beta$-dependent manner (22). Similar results were seen in the hepatoma cell lines, Hep3B and SMMC7721 (data not shown). Moreover, SB-431542, an inhibitor of the TGF- $\beta$ type I receptors (T $\beta R$-I) ALK4, -5, and -7, did not affect FHL1 modulation of reporter activity, whereas the same inhibitor fully abolished RBPMS activity (Figure 3A). Consistent with these results, FHL1 increased Smad2/3 phosphorylation in T47D cells that lack the T $\beta$ R-II and are insensitive to TGF- $\beta$ (26) (Figure 3B).
We confirmed the TGF- $\beta$ receptor-independent phosphorylation of Smad proteins induced by FHL1 with in vitro kinase assays. Treatment with the T $\beta$ R-I inhibitor, SB-431542, completely abolished the in vitro kinase activity of the RBPMS immunoprecipitate, but not of the FHL1 immunoprecipitate (Figure 3C), against Smad proteins. FHL1 immunoprecipitate phosphorylated His-Smad2(S249,254A) and His-Smad3(S423,425A) but not HisSmad2(S465,467A) and His-Smad3(S207,212A), suggesting that Ser 465 and Ser 467 in Smad2 and Ser 207 and Ser 212 in Smad3 are the sites phosphorylated by FHL1 immunoprecipitates.

CK1 $\mathrm{\delta}$ interacts with FHL1 and is required for FHL1-dependent effects on Smad proteins. While the cellular response to FHL1 expression approximates the TGF- $\beta$ response, the effects of FHL1 are independent of TGF- $\beta$ and its receptor. Therefore, we hypothesized 

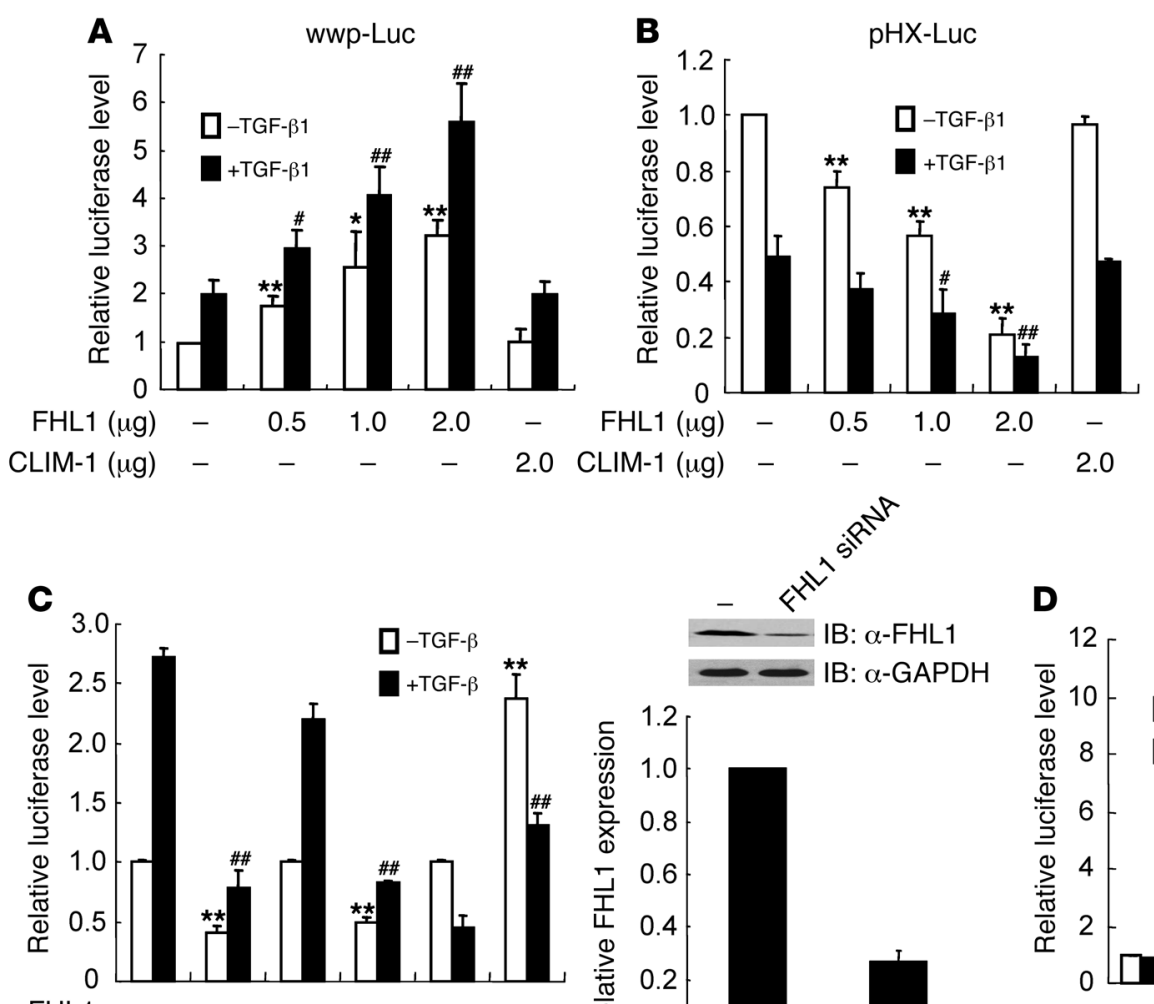

FHL1

SiRNA
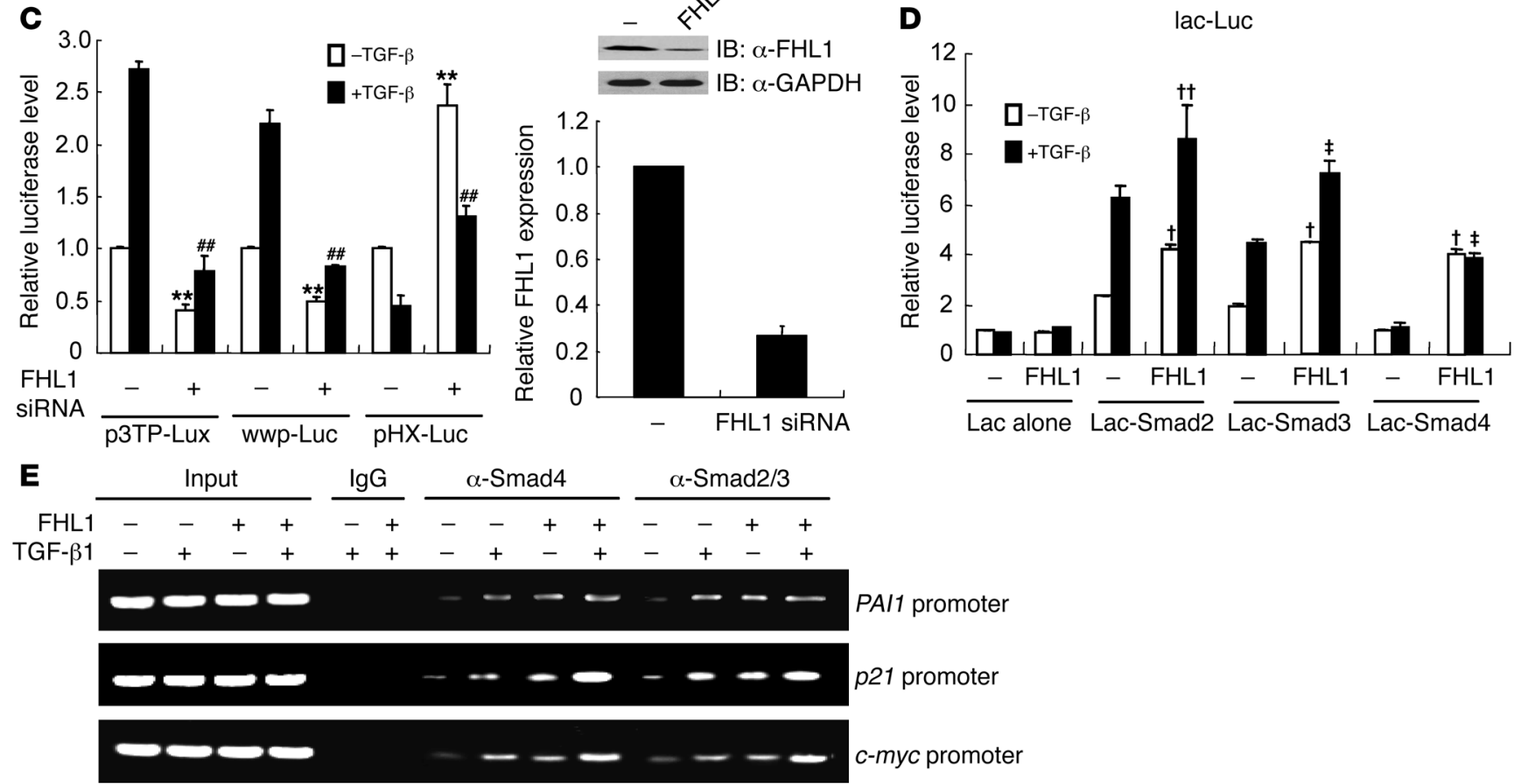

Figure 2

FHL1 regulates TGF- $\beta$-responsive transcription and the promoter occupancy of Smad proteins. (A and B) HepG2 cells were cotransfected with the TGF- $\beta$ signaling pathway reporters wwp-Luc (A) or pHX-Luc (B) and FLAG-tagged FHL1 or CLIM-1. Cells were treated with (+) or without $(-)$ TGF- $\beta 1$ and analyzed for luciferase activity. Values are mean \pm SD of 3 independent experiments. ${ }^{\star} P<0.05$, ${ }^{\star *} P<0.01$ versus empty vector without TGF- $\beta$. ${ }^{\#} P<0.05, \# \#<0.01$ versus empty vector with TGF- $\beta$. (C) HepG2 cells were cotransfected with the TGF- $\beta$ signaling pathway reporters p3TP-Lux, wwp-Luc, or pHX-Luc, and FHL1 siRNA (left panel). Values are mean \pm SD of triplicate measurements and have been repeated 3 times with similar results. ${ }^{* \star} P<0.01$ versus empty vector without TGF- $\beta$. ${ }^{\# \#} P<0.01$ versus empty vector with TGF- $\beta$. The representative immunoblot with anti-FHL1 shows specific knockdown of endogenous FHL1 by FHL1 siRNA (upper-right panel). The densitometric quantitation of the FHL1 band normalized to GAPDH from 3 independent experiments is shown (lower-right panel) (mean \pm SD). The molecular weight of GAPDH was $\sim 36 \mathrm{kDa}$. (D) HepG2 cells were cotransfected with lac-Luc, lac-Smad2, Smad3, or Smad4 and FHL1 as indicated. Values shown are mean \pm SD of 3 independent experiments. ${ }^{\dagger} P<0.01$ versus lac-Smad2, -3 , or -4 alone without TGF- $\beta$. ${ }^{\dagger t} P<0.05$ versus lac-Smad2 alone with TGF- $\beta$. $\neq P<0.01$ versus lac-Smad3 or lac-Smad4 alone with TGF- $\beta$. (E) Soluble chromatin was prepared from FHL1-transfected HepG2 cells with or without TGF- $\beta 1$ and subjected to immunoprecipitation with normal serum or indicated antibodies. Immunoprecipitated DNA was PCR amplified with primers that annealed to the proximal region of the PAl-1, p21, or c-myc promoter.

that a kinase(s) other than T $\beta$ R-I is involved in the phosphorylation of Smad2 and Smad3 by the FHL1 complex. We used the lac system to screen a protein kinase genome-wide full-length cDNA-transfection (GFC-transfection) array, consisting of 352 transfection-ready cDNA plasmids, and identified CK1 $\delta$, AURKA, and TESK1 (data not shown). The CK1 family members CK1 1 1,
CK $1 \gamma 1, \mathrm{CK} 1 \gamma 2, \mathrm{CK} 1 \gamma 3$, and CK1 $1 \varepsilon$ had no detectable affect on FHL1-mediated phosphorylation. In analyses using the p3TP-Lux and wwp-Luc reporters, only CK1 $\delta$ enhanced the FHL1-mediated activity of both reporters (Figure $4 \mathrm{~A}$ and data not shown). Moreover, FHL1 associated with CK1 $\delta$ in GST pull-down (Figure 4B) and immunoprecipitation assays (Figure 4C). CK1 $\delta$ inter- 
A

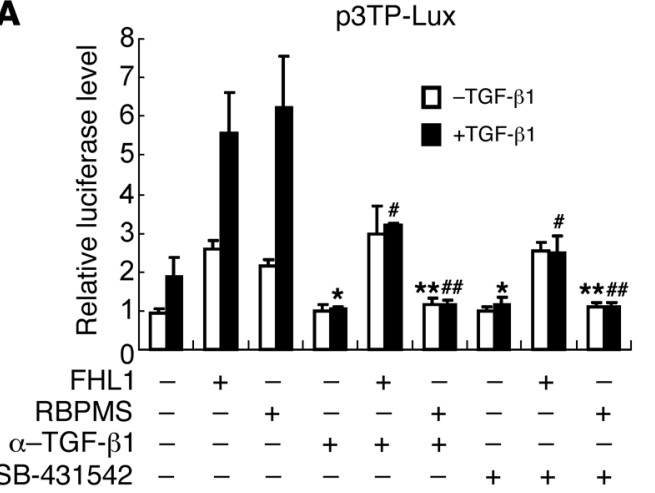

B

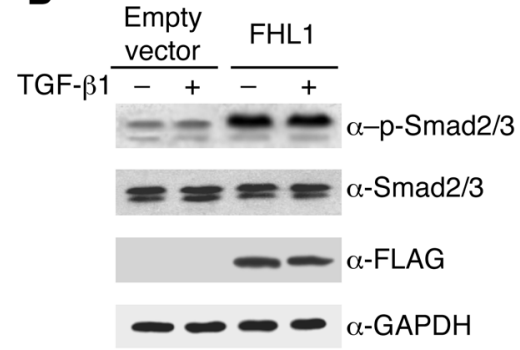

C
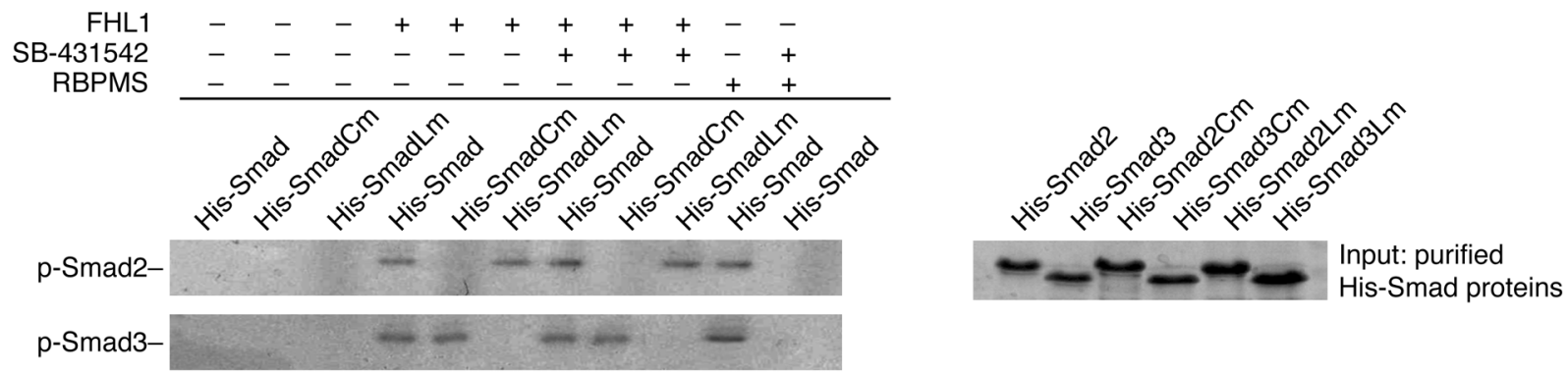

Figure 3

FHL1 regulates TGF- $\beta$-responsive transcription and Smad2/3 phosphorylation in TGF- $\beta$ - and TGF- $\beta$ receptor-independent manners. (A) HepG2 cells were cotransfected with p3TP-Lux and FHL1 or RBPMS, with or without $5 \mathrm{ng} / \mathrm{ml} \mathrm{TGF-} \beta 1,10 \mu \mathrm{g} / \mathrm{ml}$ TGF- $\beta$-neutralizing antibody $(\alpha-$ TGF- $\beta$ ), or $1 \mu \mathrm{M}$ SB-431542. Cells were analyzed as in Figure $2 \mathrm{~A}$. Values shown are mean \pm SD of triplicate measurements and have been repeated 3 times with similar results. ${ }^{\star} P<0.05$ versus empty vector with TGF- $\beta$. ${ }^{\star \star} P<0.01$ versus RBPMS without TGF- $\beta$. ${ }^{\#} P<0.05$ versus FHL1 with TGF- $\beta$. ${ }^{\#} P<0.01$ versus RBPMS with TGF- $\beta$. (B) T47D cells were transfected with FLAG-tagged FHL1 and treated with 5 ng/ml TGF- $\beta 1$. Cell lysates were analyzed by immunoblot with anti-phospho-Smad2/3 (Ser423/425) (p-Smad2/3) or anti-Smad2/3. (C) Extracts were immunoprecipitated with anti-FLAG from FLAG-tagged FHL1- or RBPMS-expressing HepG2 cells, treated with $5 \mathrm{ng} / \mathrm{ml}$ TGF- $\beta 1$ and $10 \mu M$ SB-431542. In vitro kinase assays were performed with His-Smad2, His-Smad3, His-Smad2(S465,467A) (His-Smad2Cm), His-Smad3(S423,425A) (His-Smad3Cm), His-Smad2(S249,254A) (His-Smad2Lm), or His-Smad3(S207,212A) (His-Smad3Lm) as substrate. The molecular weights were as follows: p-Smad2/3, 56 kDa/51 kDa; Smad2/3, 56 kDa/51 kDa; His-Smad2Cm, 57 kDa; His-Smad2Lm, 57 kDa; His-Smad3Cm, $\sim 52 \mathrm{kDa}$; His-Smad3Lm, 52 kDa.

acted with FHL1 only in cytoplasmic fractions of HepG2 and SMMC7721 lysates (data not shown). Sequential coimmunoprecipitation revealed that FHL1, CK1 $\delta$, and Smad proteins existed in complexes (Figure 4D). Further analysis of the interaction domain of Smad 2 and CK1 $\delta$ showed that, like T $\beta R-I$ (27), CK1 $\delta$ interacted with Smad2 $276-467$ containing the MH2 domain but not with Smad $2_{1-195}$ containing the MH1 domain or Smad2 $2_{185-286}$ containing the linker (Figure 4E).

We determined the effect of CK1 $1 \delta$ on FHL1 immunoprecipitate phosphorylation of Smad2 and Smad3 with in vitro kinase assays. Like FHL1 immunoprecipitate, CK1 $\delta$ immunoprecipitate phosphorylated His-Smad2, His-Smad2(S249,254A), His-Smad3, and His-Smad3(S423,425A) but not His-Smad2(S465,467A) or

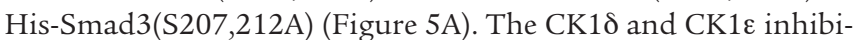
tor, IC261, fully abolished the kinase activity of CK1 $\delta$ and FHL1 immunoprecipitates. Moreover, CK1 $\delta$ directly phosphorylated Smad2 and Smad3, and FHL1 increased this activity (Figure 5B).

Given that CK1 $\delta$ phosphorylates Smad2 at its C terminus and Smad3 at its linker, while T $\beta$ R-I phosphorylates both substrates at their C-termini, we examined the effects of CK1 $1 \delta$ on T $\beta$ R-I-catalyzed C-terminal phosphorylation of Smad2/3. Immunoblotting with anti-phospho-Smad $2 / 3$ indicated that both CK $1 \delta$ and T $\beta R-I$ increased the C-terminal phosphorylation of Smad2/3 (Figure
5C). Interestingly, CK1 $\delta$ and T $\beta$ R-I additively affected C-terminal phosphorylation of Smad2/3.

To determine whether CK1 $\delta$ phosphorylates Smad3 at its linker region in vivo, HepG2 cells were cotransfected with CK1 $\delta$ and FLAGtagged Smad3 or Smad3(S207,212A). CK1 $\delta$ increased the phosphorylation of FLAG-Smad3 but not of FLAG-Smad3(S207,212A) (Supplemental Figure 3A), confirming the in vitro kinase assay results. Importantly, CK1 $\delta$ enhanced the interaction of Smad4 and FLAG-Smad3 but not FLAG-Smad3(S207,212A) (Supplemental Figure 3B). Subcellular fractionation analysis indicated that CK1 $\delta$ increased the amount of FLAG-Smad3 and-Smad4 complex formed but did not affect the formation of FLAG-Smad3(S207,212A) and Smad4 complexes in the nuclear fraction (Supplemental Figure 3C). Moreover, CK1 $\delta$ enhanced FLAG-Smad3-mediated but not FLAGSmad3(S207,212A)-mediated $p 21$ gene expression (Supplemental Figure 3D). Therefore, CK1 $\delta$ activity mimics T $\beta R$-I-mediated C-terminal phosphorylation of Smad3.

Since CK1 $\delta$ has T $\beta$ R-I-like activity and CK1 $\delta$ interacts with FHL1, we sought to determine whether FHL1 affects Smad2/3 phosphorylation, interaction of Smad $2 / 3$ with Smad 4 , and the nuclear accumulation of the Smad proteins through interaction with CK1ס. Smad2/3 phosphorylation increased in HepG2 cells stably transfected with FLAG-FHL1 (Figure 5D), while knockdown of CK1 $\delta$ in 


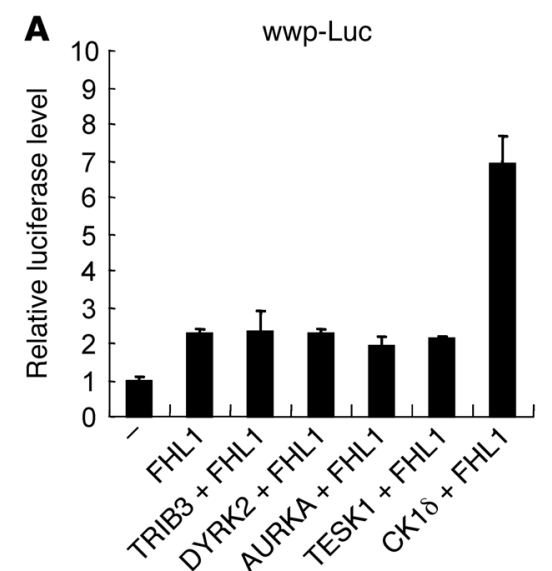

B
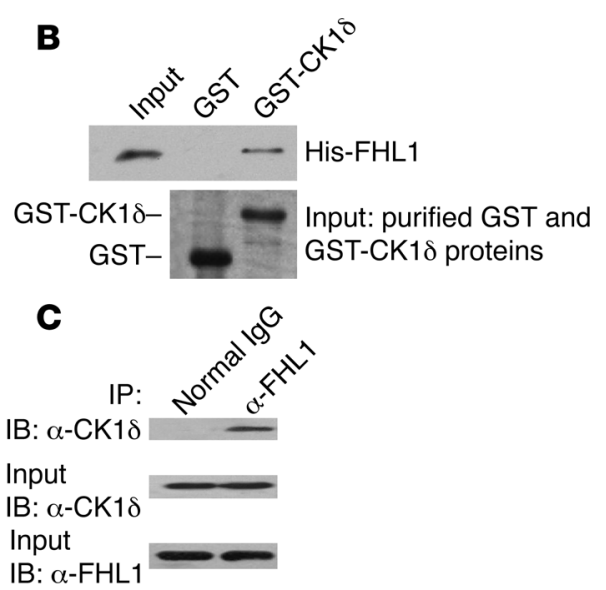

D

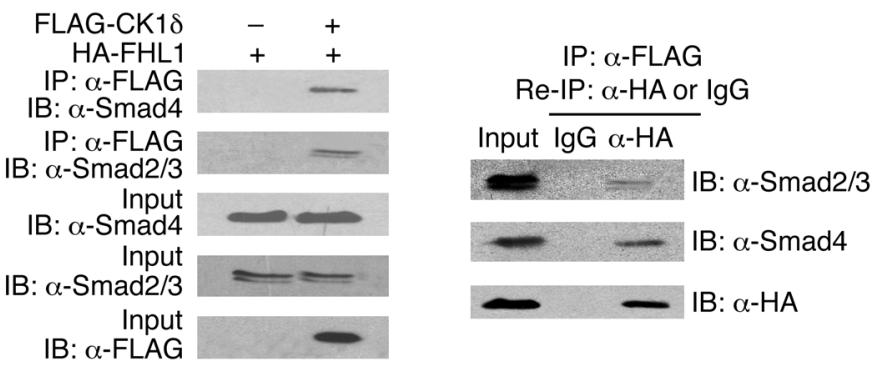

E
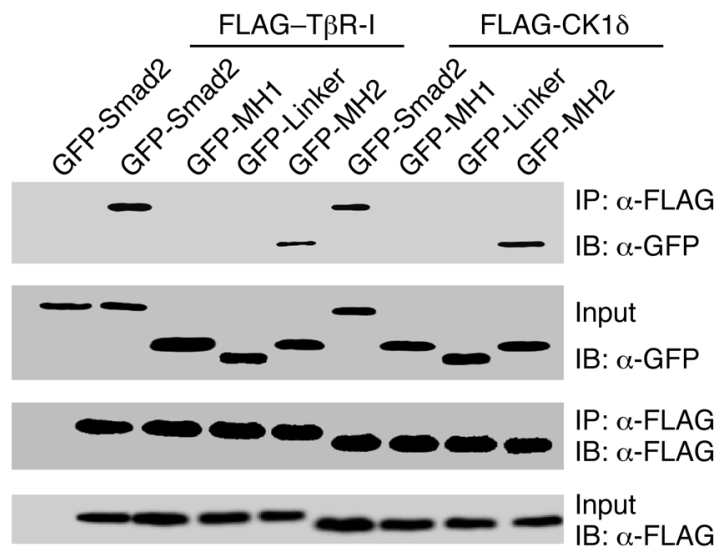

\section{Figure 4}

FHL1, CK1 $\delta$, and Smad proteins form complexes. (A) HepG2 cells were cotransfected with wwp-Luc, FHL1, and the indicated kinases, including TRIB3, DYRK2, AURKA, TESK1, and CK1 $\delta$. Twenty four hours after transfection, cells were assayed for luciferase activity. Values shown are mean \pm SD of triplicate measurements. (B) Purified GST or GST-CK1 $\delta$ was incubated with purified His-FHL1, and bound proteins were subjected to SDS-PAGE and Western blot with anti-His. (C) HepG2 cells were immunoprecipitated with anti-FHL1 or preimmune control serum, and precipitates were analyzed by immunoblot with anti-CK1ס. (D) HepG2 cells transfected with the indicated plasmids were immunoprecipitated with anti-FLAG, followed by immunoblotting with the indicated antibodies. The immune complexes were eluted with FLAG peptide and reimmunoprecipitated (Re-IP) using anti-HA or normal mouse serum. The resulting precipitates were analyzed by immunoblotting with the indicated antibodies. (E) FLAG-tagged CK1 $\delta$ or T $\beta R$-I and GFP-tagged full-length Smad2 or its deletion mutants were cotransfected into $293 T$ cells. Cell lysates were immunoprecipitated with anti-FLAG and precipitates were immunoblotted with anti-GFP. The molecular weights were as follows:

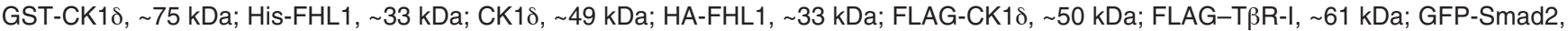
$\sim 85 \mathrm{kDa}$; GFP-MH1, $52 \mathrm{kDa}$; GFP-linker, $40 \mathrm{kDa}$; GFP-MH2, $52 \mathrm{kDa}$.

HepG2 cells reduced Smad2/3 phosphorylation. CK1 knockdown abrogated Smad2/3 phosphorylation by stably expressed FHL1 (Figure 5D). Consistent with these results, stable FHL1 expression in HepG2 cells increased the interaction of Smad2/3 and Smad4, whereas stable FHL1 expression in CK1 1 -knockdown cells failed to enhance Smad2/3 and Smad4 interaction (Figure 5E). Subcellular fractionation experiments and confocal microscopy showed that the stable FHL1 expression in HepG2 cells increased the proportion of Smad proteins in the nuclear fraction (Figure 5F and Supplemental Figure 4). However, stable FHL1 expression in CK18-knockdown cells did not promote nuclear accumulation of Smad proteins (Figure 5F), while reconstitution of CK1 $1 \delta$ expression in CK1 $\delta$-knockdown cells rescued this defect (Figure 5, D-F). Therefore, CK1ס is required for cellular responses to FHL1 expression, including phosphorylation of $S \operatorname{mad} 2 / 3$, increased interaction of $\operatorname{Smad} 2 / 3$ and Smad4, and nuclear accumulation of Smad proteins.
CK1 $\delta$ is required for FHL1-mediated TGF- $\beta$-responsive gene expression. To investigate whether the FHL1-CK1 $\delta$ interaction affects TGF- $\beta$ responsive gene transcription, HepG2 cells were cotransfected with FHL1, CK18, and either the p3TP-Lux or wwp-Luc reporter. CK1 $\delta$ or FHL1 expression increased reporter activity in a TGF- $\beta$-independent manner (Figure 6A and data not shown). Cotransfection of FHL1 and CK1 1 synergistically increased reporter gene expression (Figure 6A). Similar results were observed in the hepatoma cell lines, Hep3B and SMMC7721 (data not shown). Importantly, CK1 $\delta$ siRNA completely abolished FHL1 activation of the p3TPLux reporter (Figure 6B). As a control, and consistent with results obtained with Smad4-deficient MDA-MB-468 cells, siRNA knockdown of endogenous Smad4 in HepG2 cells also abrogated FHL1induced activation of the reporter.

To validate the reporter assay, real-time RT-PCR analysis was used to show that CK1 $\delta$ siRNA abolished FHL1 activation of 
A

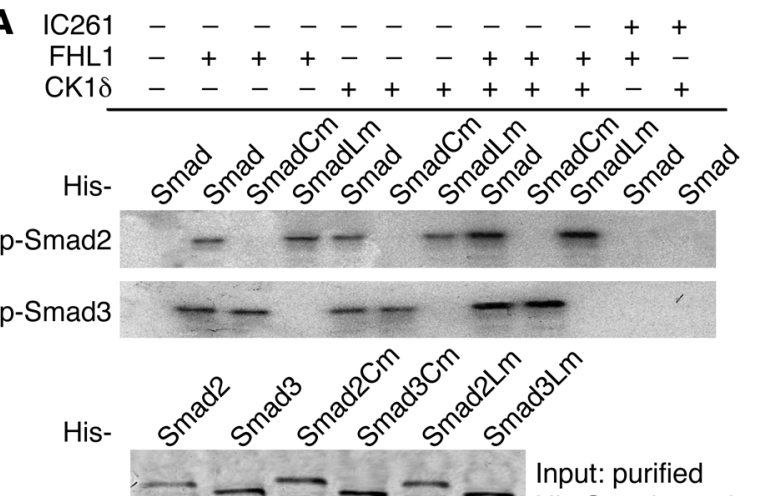

B

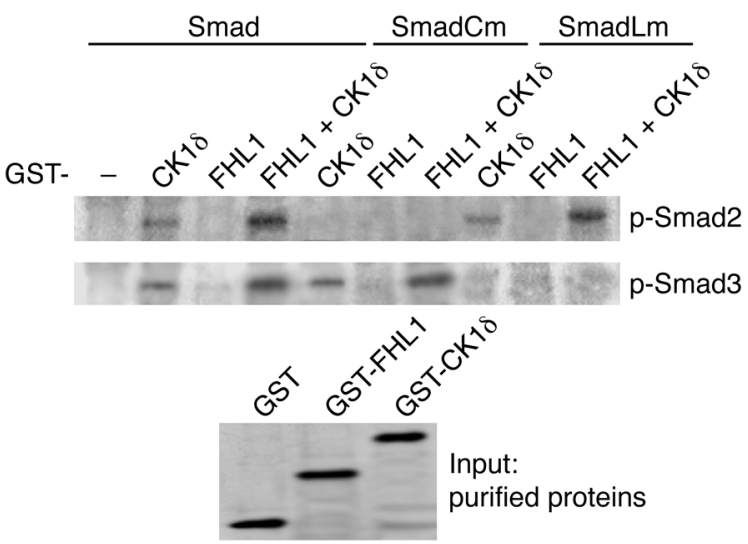

C

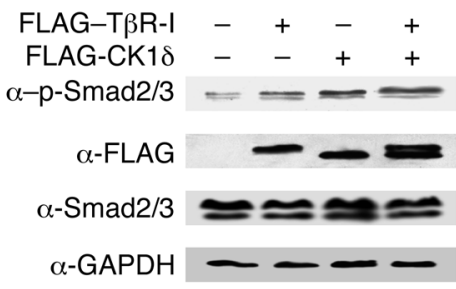

D

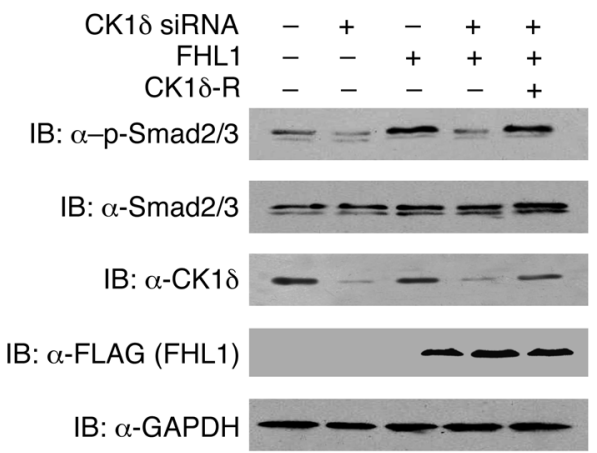

E

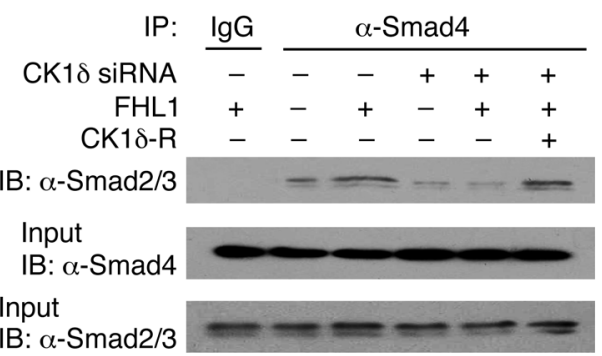

\section{Figure 5}

CK1 1 is required for FHL1 modulation of Smad2/3 phosphorylation, Smad2/3 and Smad4 interaction, and nuclear accumulation of Smad proteins.

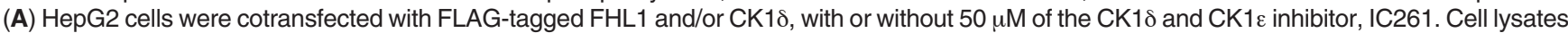
were immunoprecipitated with anti-FLAG, and in vitro kinase assays were performed using the indicated His-Smad2/3 or mutants (His-SmadCm or His-SmadLm) as substrates. (B) In vitro kinase assays with purified GST-CK1 $\delta$ and GST-FHL1 and purified His-Smad2/3 or mutants as substrates. The molecular weight of GST-FHL1 was $\sim 58 \mathrm{kDa}$. (C) HepG2 cells were transiently transfected with FLAG-tagged CK1 $\delta$ and/or T $\beta R$-I, and cell lysates were analyzed by immunoblotting with anti-phospho-Smad2/3 or anti-Smad2/3. (D) HepG2 cells stably expressing CK1 $\delta$ siRNA and FHL1 were transiently transfected with siRNA-resistant CK1 $\delta(C K 1 \delta-R)$ as indicated, and cell lysates were analyzed by immunoblotting with the indicated antibodies. Transient transfection efficiency was approximately $70 \%$. (E and F) HepG2 cells stably expressing CK1 $\delta$ siRNA and FHL1 were transiently transfected with siRNA-resistant CK1 $\delta$ as in D. (E) Cells were immunoprecipitated by anti-Smad4 or preimmune control serum, followed by immunoblotting with the indicated antibodies, or $(\mathbf{F})$ cells were fractionated and the lysates were probed with the indicated antibodies.

the TGF- $\beta$-responsive genes, $P A I-1$ and $p 21$ (Figure $6 \mathrm{C}$ and data not shown). Furthermore, stable FHL1 expression increased PAI-1 and p21 protein levels and decreased c-myc protein levels, whereas siRNA knockdown of CK1 $\delta$ reduced PAI- 1 and $\mathrm{p} 21$ protein levels and increased c-myc levels (Figure 6D). Importantly, stable FHL1 expression failed to regulate expression of these TGF- $\beta$-responsive proteins in CK $1 \delta$-knockdown cells. Similar results were obtained in SMMC7721 cells (data not shown). Therefore, CK $1 \delta$ is required for FHL1-mediated TGF- $\beta$-responsive gene expression.
FHL1, FHL2, and FHL3 inbibit hepatoma cell growth in vitro and in nude mice. Since FHL1, FHL2, and FHL3 induce TGF- $\beta$-like responses (see above and data not shown), the effects of FHL1-3 on HepG2 cell growth were examined. HepG2 cells stably transfected with FHL1, FHL2, or FHL3 grew more slowly than those transfected with empty vector (Figure 7A and data not shown), whereas HepG2 cells stably transfected with FHL1 siRNA, FHL2 siRNA, or FHL3 siRNA grew faster than those transfected with control siRNA (Figure 7B and data not shown). The observed siRNA phenotypes were rescued by siRNA-resistant FHL1, FHL2, 

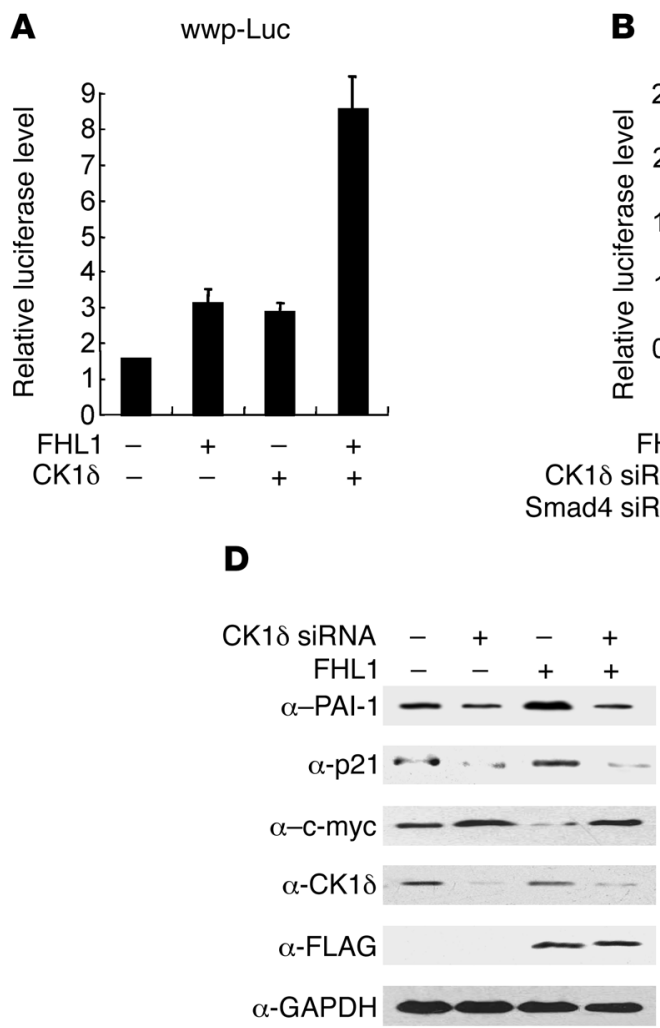

B

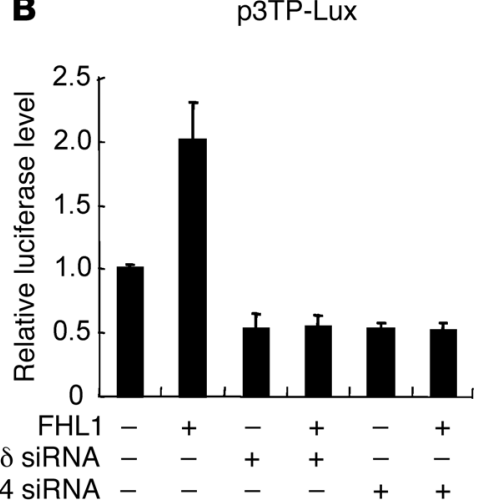

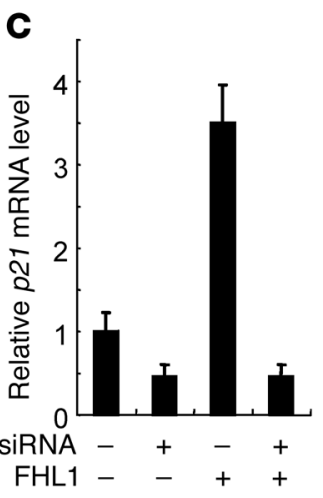

\section{Figure 6}

CK1 $\delta$ is required for FHL1-mediated TGF- $\beta$-responsive target gene expression. (A) HepG2 cells were cotransfected with wwp-Luc and CK1 $\delta$ or FHL1, individually or in combination, and analyzed for luciferase activity. Values shown are mean \pm SD of triplicate measurements. (B) Luciferase assays with HepG2 cells cotransfected with p3TP-Lux, FHL1, and the indicated siRNAs. Values shown are mean \pm SD of triplicate measurements. (C and D) HepG2 cells stably expressing CK1 $\delta$ siRNA and FLAG-tagged FHL1, individually or in combination, were used for (C) real-time RT-PCR with $p 21$ primers or (D) immunoblot with the indicated antibodies. The densitometric quantitation of PAl-1, p21, and c-myc bands normalized to GAPDH is shown at right. The molecular weights were as follows: PAl1, $52 \mathrm{kDa}$; p21, 21 kDa; c-myc, 60 kDa. Values shown are mean \pm SD of triplicate measurements and (C and D) 3 independent experiments (D).

and FHL3 constructs (Figure 7B and data not shown). Similar results were observed in the hepatoma cell lines, Hep3B and SMMC7721 (data not shown).

Next, the effects of FHL1-3 on anchorage-independent growth were tested in stable HepG2 cell lines expressing either FHL1-3 or FHL1-3 siRNAs. Overexpression of FHL1-3 decreased anchorage-independent HepG2 cell growth (Figure 7C and data not shown), whereas siRNA knockdown of endogenous FHL1-3 levels increased anchorage-independent HepG2 cell growth (Figure $7 \mathrm{D}$ and data not shown). Again, the observed siRNA effects were rescued by siRNA-resistant FHL1, FHL2, and FHL3 constructs (Figure 7D and data not shown). Similar results were seen in the hepatoma cell lines, Hep3B and SMMC7721 (data not shown).

To measure the antitumoral efficacy of FHL1, FHL2, and FHL3 in vivo, stable HepG2 cell lines expressing either FHL1-3 or the corresponding siRNAs were injected subcutaneously into nude mice. One hundred percent of injected animals developed a tumor. FHL1-3 overexpression decreased HepG2 tumor growth without noticeable side effects (Figure 7E), while siRNA knockdown of endogenous FHL1-3 expression increased HepG2 tumor growth (Figure 7F). Because they developed large tumors, nude mice inoculated with FHL1 siRNA-expressing HepG2 cells had to be sacrificed 1 week earlier than animals in the other groups. As expected, the tumors in mice inoculated with any FLAG-FHL-expressing HepG2 cell lines had increased levels of p21 and decreased levels of c-myc (Figure $7 \mathrm{G}$ ). The opposite trend was observed in tumors of mice inoculated with FHL siRNA-expressing HepG2 cells. Knockdown of any single FHL protein did not significantly change expression of the other FHL proteins (Figure 7G). These data suggest that FHL1-3 strongly suppress hepatoma cell growth, both in vitro and in vivo.

FHL1-dependent decrease of cancer cell growth requires $C K 1 \delta$ and Smad4. To examine the requirement of CK1 $\delta$ for FHL1 inhibition of cancer cell growth, HepG2 cells were sequentially and stably transfected with expression vectors for CK1 $\delta$ siRNA and FHL1. Independently, CK1 $\delta$ siRNA increased HepG2 cell growth, and stable FHL1 expression in CK1ס-knockdown cells failed to inhibit HepG2 cell growth (Supplemental Figure 5A). Reconstitution of CK1 $\delta$ expression in the knockdown cells rescued FHL1-dependent inhibition of HepG2 cell growth.

To investigate whether FHL1 inhibition of cancer growth is dependent on Smad4, Smad4-deficient breast cancer MDA-MDA-468 cells were transiently transfected with EGFP-tagged FHL1, with or without Smad4. Flow cytometric analysis indicated that ectopic FHL1 expression had little effect on cell cycle progression 
A

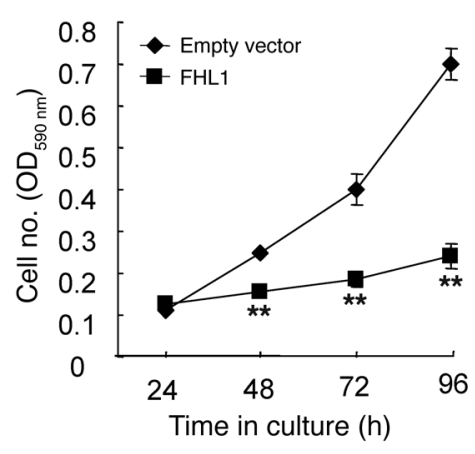

C
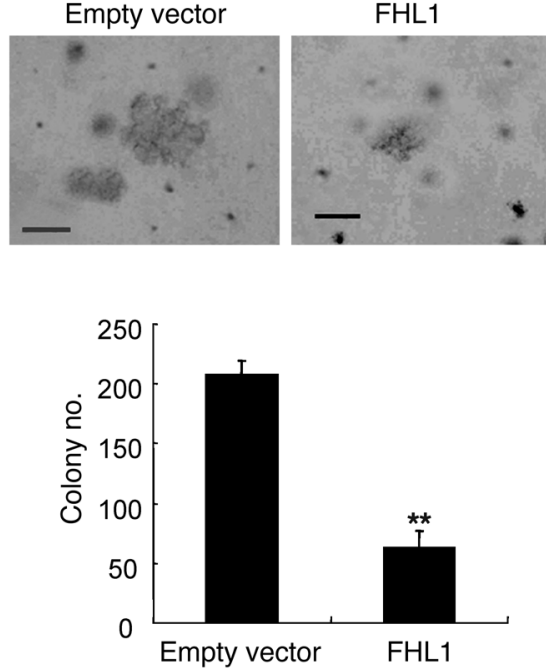

B

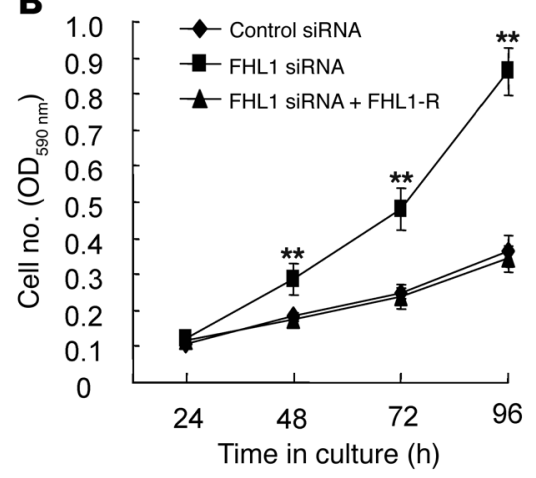

D Control siRNA FHL1 siRNA
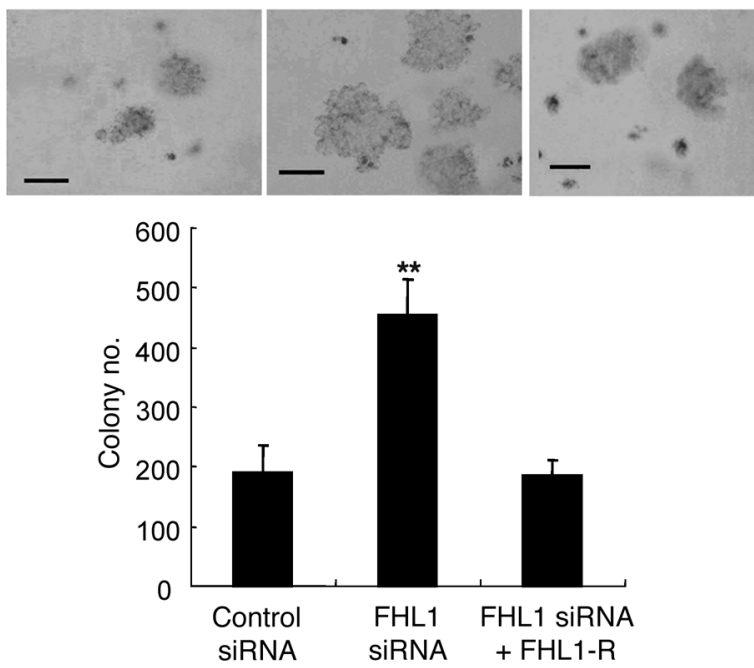

E

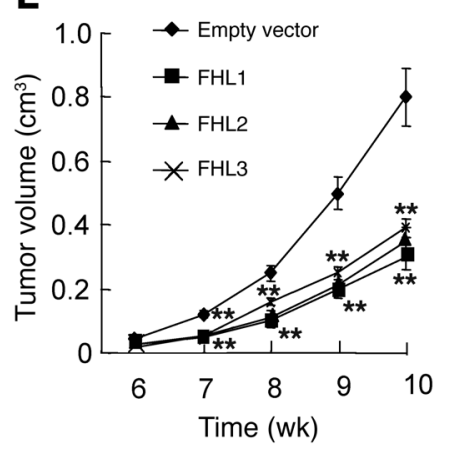

$\mathbf{F}$

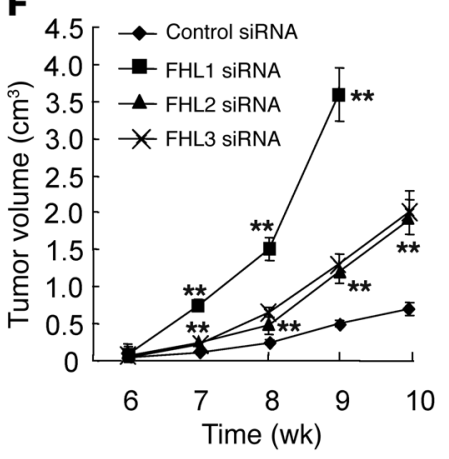

G

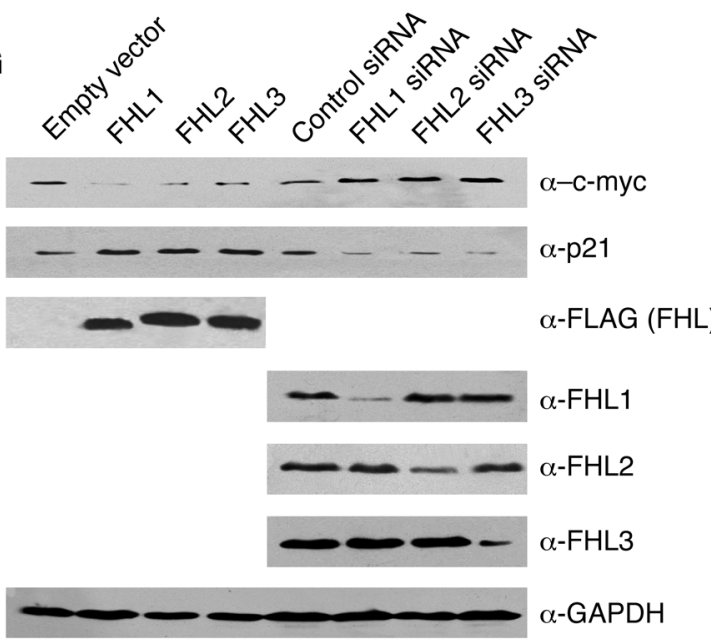

Figure 7

FHL1-3 inhibit HepG2 cell growth in vitro and in vivo. (A and B) HepG2 cells expressing FHL1 (A) or FHL1 siRNA or FHL1 siRNA plus siRNAresistant FHL1 (FHL1-R) (B) were cultured in regular medium. At specified times, cell numbers were determined by crystal violet assay. Values shown are mean \pm SD of triplicate measurements and have been repeated 3 times with similar results. ${ }^{* *} P<0.01$ versus empty vector or control siRNA. (C and D) HepG2 cells expressing FHL1 (C) or FHL1 siRNA or FHL1 siRNA plus siRNA-resistant FHL1 (D) were plated in soft agar and assayed for colony number after 3 weeks. Colonies in soft agar are shown in the photographs. Values shown are mean \pm SD of triplicate measurements and have been repeated 3 times with similar results. ${ }^{* *} P<0.01$ versus empty vector or control siRNA. Scale bar: $100 \mu \mathrm{m}$. (E and $\left.\mathbf{F}\right)$ HepG2 cells stably expressing FHL1-3 (E) or FHL1-3 siRNA (F) were injected into nude mice. At the indicated times, tumors were measured with Vernier calipers (mean $\pm \mathrm{SD} ; n=10$ ). ${ }^{* *} P<0.01$ versus empty vector or control siRNA. (G) Expression of FHL1, FHL2, and FHL3 as well as TGF- $\beta$-responsive target proteins in nude mice. Nude mice inoculated with FHL1-3 or FHL1-3 siRNA-expressing HepG2 cells were sacrificed 9 or 10 weeks after HepG2 cell injection. Excised tumors were used for immunoblotting with the indicated antibodies. The molecular weights were as follows: FHL1, 32 kDa; FHL2, 32 kDa; FHL3, 35 kDa. 
A
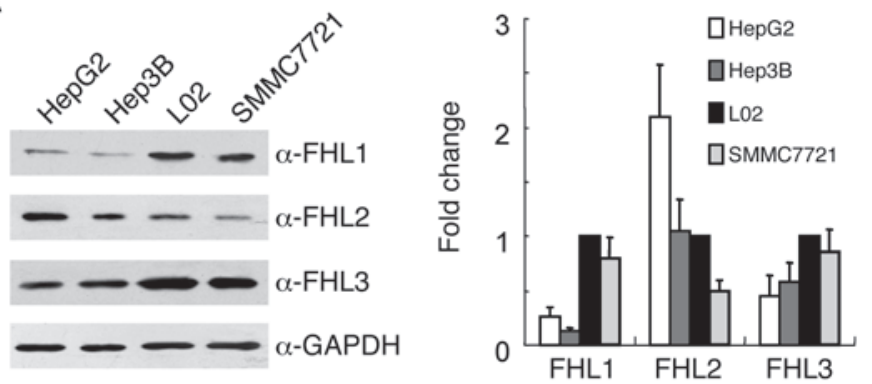

$\begin{array}{lllllllllll}\mathbf{B} & \mathrm{C} 1 & \mathrm{~N} 1 & \mathrm{C} 2 & \mathrm{~N} 2 & \mathrm{C} 3 & \mathrm{~N} 3 & \mathrm{C} 4 & \mathrm{~N} 4 & \mathrm{C} 5 & \mathrm{~N} 5\end{array}$

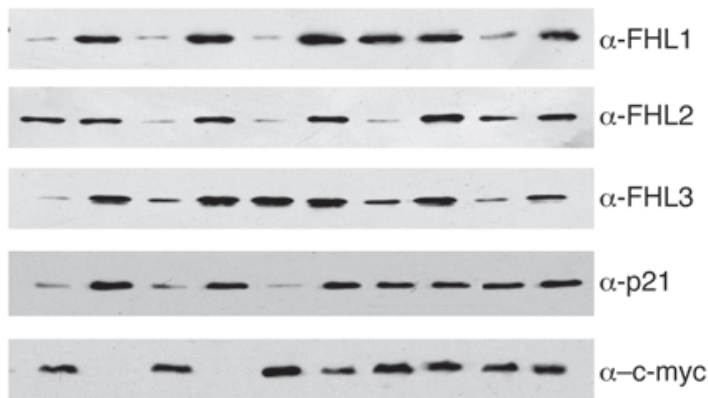

$-m-\omega=\omega-\mathrm{GAPDH}$

C
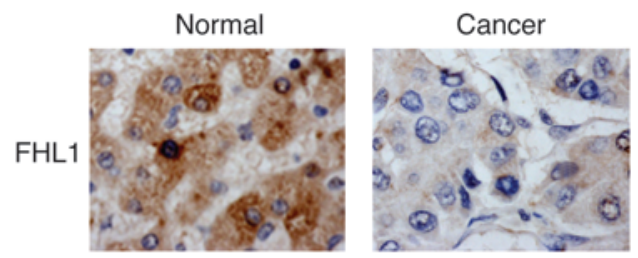

FHL2
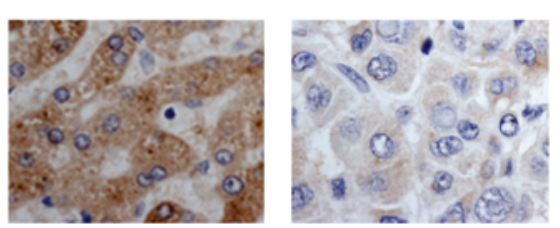

FHL3
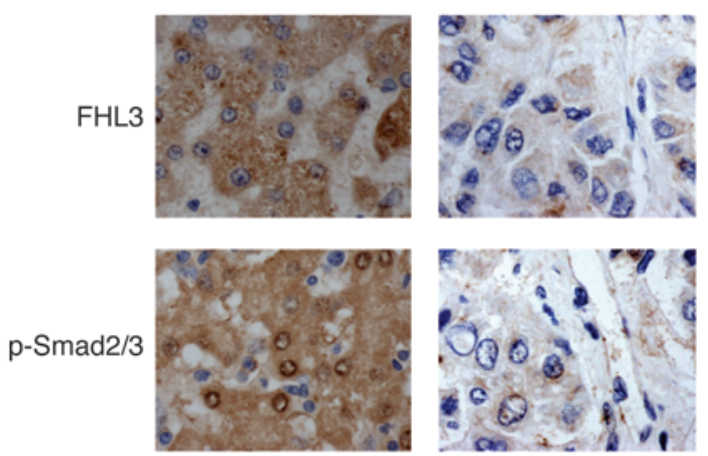
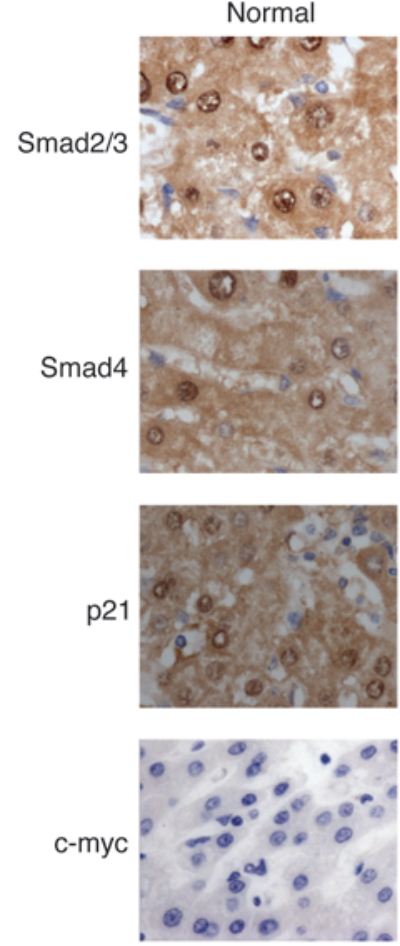

Cancer
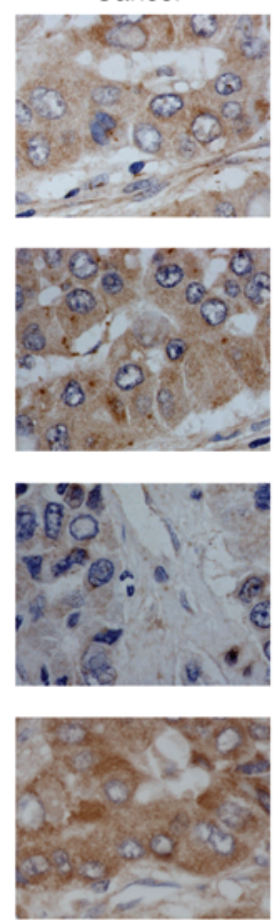

D

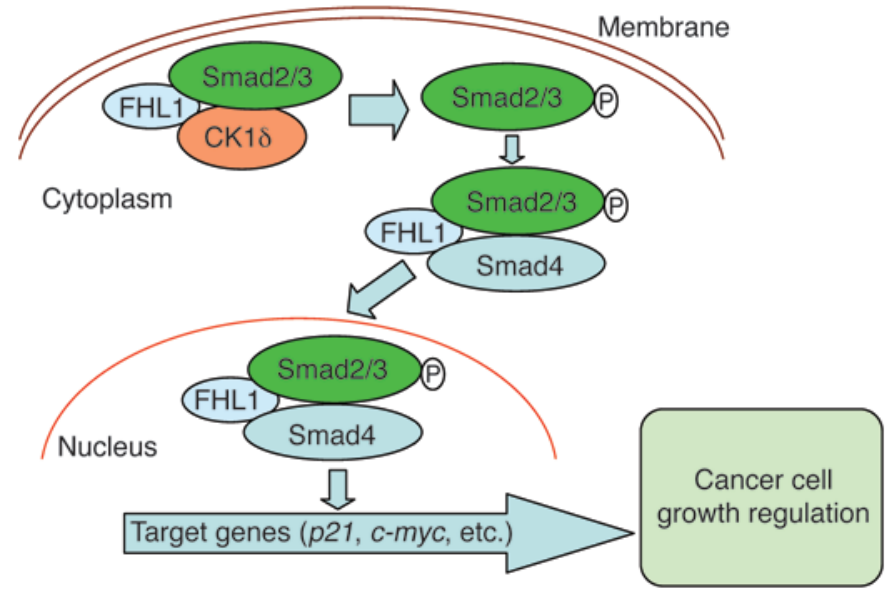




\section{Figure 8}

Expressions of FHL1-3 in human liver tumors and matched nontumor liver tissues and their correlation with TGF- $\beta$-like responses. (A) Proteins extracted from the HepG2, Hep3B, L02, and SMMC7721 cells lines were analyzed by Western blot with anti-FHL1, anti-FHL2, and anti-FHL3 (left panel). The densitometric quantitation of FHL1, FHL2, and FHL3 bands normalized to GAPDH from 3 independent experiments is shown (right panel) (mean $\pm \mathrm{SD}$ ). (B and C) Representative immunoblots (B) and immunohistochemical staining (C) of $\mathrm{FHL}$ proteins and/or the indicated proteins responsible for FHL-mediated TGF- $\beta$-like responses in human cancerous liver tissues (C1-C5) and adjacent normal liver tissues (N1-N5). Original magnification, $\times 40$. (D) Proposed model for FHL1 modulation of TGF- $\beta$-like responses. FHL1 phosphorylates cytoplasmic Smad2 and Smad3 through interaction with $\mathrm{CK} 1 \delta$, facilitating formation of Smad2/3 and Smad4 complexes and nuclear accumulation of Smad proteins. In the nucleus, Smad complexes regulate TGF- $\beta$-responsive gene transcription through interaction with FHL1.

(Supplemental Figure 5B). Smad4 overexpression alone markedly reduced the proportion of cells in $S$ phase and increased that in $G_{1}$. Intriguingly, FHL1 coexpression increased the effects of Smad4 overexpression (Supplemental Figure 5B), suggesting that FHL1 inhibits cancer cell growth at the $\mathrm{G}_{1} / \mathrm{S}$ phase.

Expression of FHL proteins in liver cancer patients and their association with TGF- $\beta$-like responses. We used Western blot analysis to examine the expression of FHL1, FHL2, and FHL3 proteins in 3 different hepatoma cell lines (HepG2, Hep3B, and SMMC7721) and 1 immortalized normal human hepatocyte line (LO2). For each FHL protein, we detected a single band of the predicted molecular mass of approximately $32 \mathrm{kDa}$ in all cell lines tested (Figure $8 \mathrm{~A})$. In most cases, hepatoma cells expressed reduced FHL protein levels than $\mathrm{LO} 2$ cells.

We next examined the expression of FHL proteins and their targets, p21 and c-myc, in liver cancer patients and matched healthy controls. Of 20 liver tumors, 16, 15, and 16 expressed lower levels of FHL1, FHL2, and FHL3 proteins, respectively, than control livers (Figure 8B). Reduced FHL protein levels correlated with reduced p21 levels and increased c-myc levels.

We used immunohistochemistry to detect FHL protein expression and the associated TGF- $\beta$-like responses in 115 pairs of HCC tumors and matched nontumor liver tissues. In agreement with the Western blot data, 93.0\% (107/115), 88.7\% (102/115), and $92.2 \%(106 / 115)$ of healthy livers expressed FHL1, FHL2, and FHL3, respectively, while only $23.5 \%$ (27/115), 37.4\% (43/115), and $28.7 \%(33 / 115)$ of cancerous tissues stained positive for these FHL proteins (Figure 8C). Expression of FHL1-3 positively correlated with Smad $2 / 3$ phosphorylation $(P<0.05)$, nuclear accumulation of Smad2-4 $(P<0.05)$, and p21 expression $(P<0.05)$ and negatively correlated with $\mathrm{c}-\mathrm{myc}$ expression $(P<0.05)$ (Figure $8 \mathrm{C}$ and Table $1)$. Together, these data strongly suggest important pathological roles of FHL1, FHL2, and FHL3 in HCC.

\section{Discussion}

We demonstrated for what we believe to be the first time that, independent of TGF- $\beta$ and TGF- $\beta$ receptor, FHL1-3 physically and functionally interact with Smad2-4. Like Smads, FHL proteins localize to both the cytoplasm and the nucleus. FHL proteins enhanced Smad2/3 phosphorylation, promoted the interaction of Smad2/3 with Smad4, and influenced the nuclear accumulation of Smad proteins. Similar to the TGF- $\beta$ response, FHL pro- teins ultimately increased expression of growth inhibitor genes, such as the CDK inhibitor $p 21$, and decreased expression of the growth-promoting gene $c-m y c$. Through interaction with CK1 $\delta$, FHL1 phosphorylated Smad2 at the C terminus and Smad3 at the linker region, whereas the TGF- $\beta$ receptor phosphorylated both Smad 2 and Smad 3 at the C terminus. Thus, FHL1 may regulate additional targets that were not affected by TGF- $\beta$. The fact that FHL proteins were recruited to TGF- $\beta$-responsive promoters suggests that FHL proteins function both in the cytoplasm and nucleus (Figure 8D). Smad4, the central mediator of TGF- $\beta$ signaling, and CK1 $\delta$ are required for FHL function. FHL1, FHL2, and FHL3 inhibited, to varying degrees, anchorage-dependent and -independent hepatoma cell growth in vitro and tumor formation in nude mice, through upregulation of $\mathrm{p} 21$ and downregulation of c-myc. Moreover, FHL1-3 were downregulated in liver cancer patients and correlated with TGF- $\beta$-like responses. Our observations that coexpression of the FHL proteins in hepatoma cells may additively affect TGF- $\beta$-like responses and that knockdown of any single FHL protein did not affect the levels of the other FHL proteins suggest that no functional redundancy or compensation among the closely related FHL proteins exists. Recently, a mutated FHL1 gene was found in X-linked dominant scapuloperoneal myopathy and reduced of body myopathy (28, 29). It will be interesting to investigate $F H L$ gene mutations in human tumors such as liver tumors. Taken together, our data suggest that FHL proteins may be useful molecular targets for cancer therapy, especially liver cancer therapy.

Smad2/3 and Smad4 complexes have been thought to exert transcriptional activity in TGF- $\beta$ - and TGF- $\beta$ receptor-dependent manners. However, we demonstrated that Smad proteins regulate transcription through interaction with FHL proteins in a TGF- $\beta$ and TGF- $\beta$ receptor-independent manner. First, FHL proteins modulated Smad-mediated transcriptional activity independent of TGF- $\beta$, and addition of a TGF- $\beta$-neutralizing antibody did not affect FHL-induced Smad trans-activation. Second, SB-431542, an inhibitor of the T $\beta$ R-I ALK4, -5, and -7, had no effect on FHLinduced Smad trans-activation. Third, FHL1 enhanced Smad2/3 phosphorylation in T47D cells that lack T $\beta \mathrm{R}-\mathrm{II}$ and are insensitive to TGF- $\beta$. Finally, FHL1 immunoprecipitate phosphorylated Smad2/3 in the presence of the T $\beta$ R-I inhibitor, SB-431542.

FHL1 cannot directly phosphorylate Smad2 and Smad3, and we identified $\mathrm{CK} 1 \delta$ as the protein responsible for kinase activity in FHL1 immunoprecipitates. Similar to FHL1, CK1 $\delta$ increased p21 expression and decreased c-myc expression, although we cannot exclude the possibility that FHL1 and CK1 $\delta$ target different genes. We conclude that CK1 $\delta$ is required for FHL1 function, because knockdown of CK1 $\delta$ completely abolished FHL1-mediated TGF- $\beta$ like responses. Thus, we identified what we believe to be a novel TGF- $\beta$-like signaling pathway that is important for Smad $2 / 3$ phosphorylation, Smad2/3 and Smad 4 interaction, Smad protein nuclear accumulation, and cancer cell growth regulation (Figure 8D).

\section{Methods}

Plasmids, siRNAs, and transfection. The p3TP-Lux (30) (gift of Joan Massague, Memorial Sloan-Kettering Cancer Center, New York, New York, USA), pHX-Luc, p15-Luc (31) (gifts of Aristidis Moustakas, Ludwig Institute for Cancer Research, Uppsala, Sweden), wwp-Luc (32) (gift of Bert Vogelstein, Johns Hopkins University School of Medicine, Baltimore, Maryland, USA), and lacLuc (33) reporter constructs and Smad2, Smad3, and Smad4 (22) expression vectors were described previously. Mammalian expression vectors encoding 


\section{Table 1}

FHL1-3 protein expression correlates with TGF- $\beta$-like responses

\begin{tabular}{|c|c|c|c|c|c|c|c|c|c|}
\hline & FHL1+ $^{+}$ & FHL1 $^{-}$ & $P A$ & FHL2+ $^{+}$ & FHL2- & $P A$ & $\mathrm{FHL3}^{+}$ & FHL3- & $P A$ \\
\hline p-Smad2/3+ & 92 & 29 & $<10^{-4}$ & 84 & 37 & 0.035 & 91 & 30 & $<10^{-4}$ \\
\hline p-Smad2/3- & 42 & 67 & $<10^{-4}$ & 61 & 48 & 0.035 & 48 & 61 & $<10^{-4}$ \\
\hline Nuclear Smad2/3+ & 80 & 40 & 0.007 & 87 & 33 & 0.002 & 82 & 38 & 0.011 \\
\hline Nuclear Smad2/3- & 54 & 56 & 0.007 & 58 & 52 & 0.002 & 57 & 53 & 0.011 \\
\hline Nuclear Smad4+ & 88 & 35 & $<10^{-4}$ & 89 & 34 & 0.002 & 86 & 37 & 0.011 \\
\hline Nuclear Smad4- & 46 & 61 & $<10^{-4}$ & 56 & 51 & 0.002 & 53 & 54 & 0.002 \\
\hline p21+ & 122 & 25 & $<10^{-4}$ & 112 & 35 & $<10^{-4}$ & 108 & 39 & $<10^{-4}$ \\
\hline $\mathrm{p} 21^{-}$ & 12 & 71 & $<10^{-4}$ & 33 & 50 & $<10^{-4}$ & 31 & 52 & $<10^{-4}$ \\
\hline $\mathrm{c}-\mathrm{myc}^{+}$ & 59 & 84 & $<10^{-4}$ & 74 & 69 & $<10^{-4}$ & 64 & 79 & $<10^{-4}$ \\
\hline c-myc- & 75 & 12 & $<10^{-4}$ & 71 & 16 & $<10^{-4}$ & 75 & 12 & $<10^{-4}$ \\
\hline
\end{tabular}

The numbers in the second, third, fifth, sixth, eighth, and ninth columns represent the numbers of clinical samples showing FHL1-3 expression. ${ }^{\mathrm{A} C o r r e l a-}$ tions between FHL1, FHL2, and FHL3 expression and TGF- $\beta$-like responses were determined by $\chi^{2}$ tests. $P$ values of the $\chi^{2}$ tests are listed.

FLAG-, HA-, or lac repressor-tagged (lac-tagged) fusion proteins were constructed by inserting PCR-amplified fragments into pcDNA3 (Invitrogen) or pIRESpuro2 (Clontech) linked to FLAG, HA, or lac at the amino terminus. EGFP-tagged fusion protein constructs were made by inserting a PCR-amplified cDNA fragment into pEGFP-C1 (Clontech). Plasmids encoding GSTand His-fusion proteins were prepared by cloning a PCR-amplified sequence into pGEX-KG (Amersham Pharmacia Biotech) and pET28a (Novagen), respectively. For yeast 2-hybrid assay, the bait plasmid, pGBKT7-Smad4 $260-514$, was generated by inserting the corresponding cDNA fragment into pGBKT7 (Clontech). The cDNA target sequences of siRNAs for FHL1, FHL2, FHL3, Smad4, and CK1 $\delta$ were AAGGAGGTGCACTATAAGAAC, AACTGCTTCTGTGACTTGTAT, AAGTACATCCAGACAGACAGC, AAGATCTACCCAAGTGCATAT, and AAATGATCAGTCGCATCGAAT, respectively, and were inserted into pSilencer2.1-U6neo (Ambion). Expression vectors for siRNA-resistant FHL1, FHL2, FHL3, or CK1 $\delta$ were generated by recombinant PCR, with a silent mutation in the $3^{\prime}$ nucleotide of a codon in the middle of the siRNA binding site. Cloning details are available in Supplemental Methods. All cell lines were obtained from the ATCC but the human breast cancer cell line MDA-MB-468, which was a kind gift of Yeguang Chen (Tsinghua University, Beijing, China). Transfections were performed using Lipofectamine 2000 (Invitrogen). After transfection, cells were treated with $5 \mathrm{ng} / \mathrm{ml} \mathrm{TGF}-\beta 1$ for 20 hours, with or without TGF- $\beta$-neutralizing antibody (R\&D Systems), in medium containing $0.5 \% \mathrm{FBS}$ or in serum-free medium. If necessary, cells were treated with the T $\beta$ R-I inhibitor, SB-431542, and the CK1 $\delta$ and CK1 $\varepsilon$ inhibitor, IC261. Luciferase and $\beta$-galactosidase activities were determined as described previously (34). For stable transfections, transfected cells were selected in $500 \mu \mathrm{g} / \mathrm{ml} \mathrm{G} 418$ (Invitrogen) or $0.1 \mu \mathrm{g} / \mathrm{ml}$ puromycin for approximately 2 months. Pooled clones or individual clones were screened by Western blot and produced similar results.

Yeast 2-bybrid screens. The Matchmaker 2-hybrid system (Clontech) was used to isolate proteins that interacted with the Smad4 bait protein. The bait plasmid and a mammary cDNA library were sequentially transformed into AH109 yeast cells as previously described (22).

GST pull-down assay. GST- and His-fusion proteins were expressed and purified, according to the manufacturers' instructions (Amersham Pharmacia and Qiagen). ${ }^{35}$ S-labeled Smad2, Smad3, Smad4, or purified HisSmad4 were incubated with GST fusion protein bound to glutathioneSepharose beads (Amersham Pharmacia), and the adsorbed proteins were analyzed as previously described (35).

Coimmunoprecipitation. Cells were transfected and lysed in lysis buffer. Subcellular fractionation and coimmunoprecipitation were performed as previously described (22).
ChIP. ChIP assays were performed as described previously (36). The following primers were used: PAI-1 promoter sense, $5^{\prime}$-CTCCACAGTGACCTGGTTCGCCA-3'; PAI-1 promoter antisense, 5'-GATGTGGGCAGGAAATAGATGA-3'; PAI-1 upstream sense, 5'-GGGACATCTAGCTATGTCTAG-3'; PAI-1 upstream antisense, 5'-TCAGCTAGGCATGGTGGTGCGT-3'; p21 promoter sense (37), 5'-GTGGCTCTGATTGCCTTTCTG-3'; p21 promoter antisense, 5'-CAGCCCTGTGGCAAGGATCC-3'; p21 upstream sense, 5'-GGATCCCTGTAGAGATGCTCAG-3'; p21 upstream antisense, 5'-ATCTGGTTGGCATCATCTCGCTG-3'; c-myc promoter sense (38), 5'-GGTCTGGACGGCTGAGGACCCCCG-3'; c-myc promoter antisense, 5'-CTCTCGCTGGAATTACTACAGCG-3'; c-myc upstream sense, 5'-GCTTGTTTGGCCGTTTTAGGGT-3'; c-myc upstream antisense, 5'-CGTCCGAGGTGCAAGGTTTCC-3'.

Kinase assays. Immune complexes or purified GST-FHL1 and GSTCK1 $\delta$ were incubated with purified His-Smad2 or -Smad3 or their mutants in kinase buffer (50 mM HEPES, $10 \mathrm{mM} \mathrm{MgCl}_{2}, 1 \mathrm{mM}$ DTT, $2.5 \mathrm{mM}$ EGTA, $0.1 \mathrm{mM} \mathrm{Na}_{3} \mathrm{VO}_{3}, 1 \mathrm{mM} \mathrm{NaF}$ ), containing $\gamma^{-32} \mathrm{P}$-ATP for 30 minutes at $37^{\circ} \mathrm{C}$. The reaction products were analyzed by SDS-PAGE and autoradiography.

Real-time RT-PCR. Total RNA was isolated using TRIzol Reagent (Invitrogen) and reverse transcribed using SuperScript II Reverse Transcriptase (Invitrogen). Real-time PCR was performed with PAI-1-, p21-, and GAPDH-specific primers as described previously $(39,40)$.

Confocal microscopy. Cells on glass coverslips were fixed with $1.6 \%$ paraformaldehyde for 30 minutes, permeabilized with $0.2 \%$ Triton X-100 for 5 minutes, and blocked in $1 \%$ normal goat serum for 1 hour. The coverslips were incubated with rabbit anti-FHL1 (Proteintech), rabbit anti-Smad2/3 (Upstate), or mouse anti-Smad4 (Santa Cruz Biotechnology Inc.) and then were incubated with goat anti-rabbit IgG (Santa Cruz Biotechnology Inc.) or goat anti-mouse IgG (Santa Cruz Biotechnology Inc.) secondary antibodies. Nuclei were counterstained with $0.2 \mu \mathrm{g} / \mathrm{ml}$ DAPI. Confocal images were collected using a Radiance 2100 confocal microscope (Bio-Rad).

Clinical samples. Liver tumor samples and adjacent noncancerous tissues were obtained from the China PLA General Hospital with the informed consent of patients and with approval for experiments from the China PLA General Hospital and Beijing Institute of Biotechnology. Immunoblot analysis was performed with anti-FHL1 (Proteintech), anti-FHL2 (Santa Cruz Biotechnology Inc.), anti-FHL3 (Proteintech), anti-p21 (Santa Cruz Biotechnology Inc.), and anti-c-myc (Santa Cruz Biotechnology Inc.). Immunohistochemistry was performed as previously described (35). Rabbit anti-FHL1, goat anti-FHL2, rabbit anti-FHL3, goat anti-phospho- 
Smad2/3 (Ser 423/425) (Santa Cruz Biotechnology Inc.), rabbit antiSmad2/3 (Upstate), mouse anti-Smad4 (Santa Cruz Biotechnology Inc.), rabbit anti-p21 (Santa Cruz Biotechnology Inc.), or mouse anti-c-myc (Santa Cruz Biotechnology Inc.) were used as primary antibodies.

Cell proliferation and cell cycle assays. Cell proliferation was analyzed by crystal violet assay as described previously (35). Cell cycle analysis was determined by flow cytometry (33). Briefly, cells were fixed in $70 \%$ ethanol, washed in PBS, and incubated with RNase A $(0.2 \mathrm{mg} / \mathrm{ml})$ in PBS. Propidium iodide was added, and samples were analyzed on a FACSCalibur Flow Cytometer (Becton Dickinson).

Anchorage-independent growth assay. Cells $\left(2 \times 10^{4}\right)$ were plated on $6-\mathrm{cm}$ plates, with a bottom layer of $0.7 \%$ low-melting-temperature agar in DMEM and a top layer of $0.25 \%$ agar in DMEM. Colonies were scored after 3 weeks of growth.

In vivo tumor growth. Animal studies were approved by the Institutional Animal Care Committee of Beijing Institute of Biotechnology. Sevenweek-old male SCID mice were subcutaneously inoculated in the right flank with $1 \times 10^{7}$ hepatoma cells, which were resuspended in PBS $(n=10)$. Growth was recorded by caliper measurements at indicated times. Excised tumors were weighed, and portions were frozen in liquid nitrogen or fixed in $4 \%$ paraformaldehyde.

1. Johannessen, M., Møller, S., Hansen, T., Moens, U., and van Ghelue, M. 2006. The multifunctional roles of the four-and-a-half-LIM only protein FHL2. Cell. Mol. Life Sci. 63:268-284.

2. Samson, T., et al. 2004. The LIM-only proteins FHL2 and FHL 3 interact with $\alpha$ - and $\beta$-subunits of the muscle $\alpha 7-\beta 1$ integrin receptor. J. Biol. Chem. 279:28641-28652.

3. Coghill, I.D., et al. 2003. FHL3 is an actin-binding protein that regulates $\alpha$-actinin-mediated actin bundling: FHL3 localizes to actin stress fibers and enhances cell spreading and stress fiber disassembly. J. Biol. Chem. 278:24139-24152.

4. Muller, J.M., et al. 2000. FHL2, a novel tissue-specific coactivator of the androgen receptor. EMBOJ. 19:359-369.

5. Martin, B., et al. 2002. The LIM-only protein FHL2 interacts with $\beta$-catenin and promotes differentiation of mouse myoblasts. J. Cell. Biol. 159:113-122.

6. Labalette, C., Renard, C.A., Neuveut, C., Buendia, M.A., and Wei, Y. 2004. Interaction and functional cooperation between the LIM protein FHL2, CBP/ p300 and $\beta$-catenin. Mol. Cell. Biol. 24:10689-10702.

7. Hill, A.A., and Riley, P.R. 2004. Differential regulation of Hand1 homodimer and Hand1-E12 heterodimer activity by the cofactor FHL2. Mol. Cell. Biol. 24:9835-9847.

8. Dye, B.T., and Patton, J.G. 2001. An RNA recognition motif (RRM) is required for the localization of PTB-associated splicing factor (PSF) to subnuclear speckles. Exp. Cell Res. 263:131-144.

9. Chan, K.K., et al. 2000. Protein-protein interaction of FHL2, a LIM domain protein preferentially expressed in human heart, with hCDC47. JCell. Biochem. 76:499-508.

10. Lange, S., et al. 2002. Subcellular targeting of metabolic enzymes to titin in heart muscle may be mediated by DRAL/FHL-2. J. Cell. Sci. 115:4925-4936.

11. Shen, Y., Jia, Z., Nagele, R.G., Ichikawa, H., and Goldberg, G.S. 2006. SRC uses Cas to suppress Fhl1 in order to promote nonanchored growth and migration of tumor cells. Cancer Res. 66:1543-1552.

12. Scholl, F.A., McLoughlin, P., Ehler, E., de Giovanni, C., and Schäfer, B.W. 2000. DRAL is a p53-responsive gene whose four and a half LIM domain protein product induces apoptosis. J. Cell Biol. 151:495-506.

13. Wang, J., et al. 2007. Suppression of FHL2 expression induces cell differentiation and inhibits gastric and colon carcinogenesis. Gastroenterology.

Statistics. Differences between variables were assessed by $\chi^{2}$ analysis or 2 -tailed Student's $t$ test. Statistical calculations were performed using SPSS 13.0. $P$ values of less than 0.05 were considered statistically significant.

\section{Acknowledgments}

We thank Aristidis Moustakas, Bert Vogelstein, and Joan Massague for plasmids and Yeguang Chen for MDA-MB-468 cells. This work was supported by Key Technologies R\&D Program (2002BA711A02-5, 2007AA02Z113, and 2006BAI23B01), Major State Basic Research Development Program (2006CB943501 and 2007CB914603), National Natural Science Foundation (30530320, 30625035, 30870499, 30500091, and 30600223), and Medicine and Health Research Foundation of PLA (06J021).

Received for publication April 14, 2008, and accepted in revised form November 19, 2008.

Address correspondence to: Qinong Ye, Beijing Institute of Biotechnology, 27 Tai-Ping Lu Rd., Beijing 100850, People's Republic of China. Phone: (8610) 6818-0809; Fax: (8610) 6824-8045; E-mail: yeqn@yahoo.com.

132:1066-1076.

14. Massagué, J., Seoane, J., and Wotton, D. 2005. Smad transcription factors. Genes Dev. 19:2783-2810.

15. Moustakas, A., Souchelnytskyi, S., and Heldin, C.H. 2001. Smad regulation in TGF- $\beta$ signal transduction. J. Cell Sci. 114:4359-4369.

16. ten Dijke, P., Goumans, M.J., Itoh, F., and Itoh, S. 2002. Regulation of cell proliferation by Smad proteins. J. Cell Physiol. 191:1-16.

17. Chen, C.R., Kang, Y., Siegel, P.M., and Massagué, J. 2002. E2F4/5 and p107 as Smad cofactors linking the TGF $\beta$ receptor to c-myc repression. Cell. 110:19-32.

18. Wotton, D., Lo, R.S., Lee, S., and Massagué, J.A. 1999. Smad transcriptional corepressor. Cell. 97:29-39.

19. Zhang, Y., Feng, X.H., and Derynck, R. 1998. Smad3 and Smad4 cooperate with c-Jun/c-Fos to mediate TGF- $\beta$-induced transcription. Nature. 394:909-913.

20. Graves, P.R., and Roach, P.J. 1995. Role of COOHterminal phosphorylation in the regulation of casein kinase Iס. J. Biol. Chem. 270:21689-21694.

21. Wu, L., et al. 2003. Smad4 as a transcription corepressor for estrogen receptor $\alpha$. J. Biol. Chem. 278:15192-15200.

22. Sun, Y., et al. 2006. Potentiation of Smad-mediated transcriptional activation by the RNA-binding protein RBPMS. Nucleic Acids Res. 34:6314-6326.

23. Shioda, T., et al. 1998. Transcriptional activating activity of Smad4: roles of SMAD hetero-oligomerization and enhancement by an associating transactivator. Proc Natl Acad Sci. U. S. A. 95:9785-9790.

24. Wu, J.W., Fairman, R., Penry, J., and Shi, Y. 2001. Formation of a stable heterodimer between Smad2 and Smad4. J. Biol. Chem. 276:20688-20794.

25. de Winter, J.P., et al. 1997. DPC4 (SMAD4) mediates transforming growth factor- $\beta 1$ (TGF- $\beta 1$ ) induced growth inhibition and transcriptional response in breast tumour cells. Oncogene. 14:1891-1899.

26. Fanayan, S., Firth, S.M., Butt, A.J., and Baxter, R.C. 2000. Growth inhibition by insulin-like growth factor-binding protein-3 in T47D breast cancer cells requires transforming growth factor- $\beta$ (TGF- $\beta$ ) and the type II TGF- $\beta$ receptor. J. Biol. Chem. 275:39146-39151.

27. Massague, J., and Wotton, D. 2000. Transcriptional control by the TGF- $\beta /$ Smad signaling system. ЕМВО J. 19:1745-1754.

28. Quinzii, C.M., et al. 2008. X-linked dominant scapuloperoneal myopathy is due to a mutation in the gene encoding four-and-a-half-LIM protein 1. Am. J. Hum. Genet. 82:208-213.

29. Schessl, J., et al. 2008. Proteomic identification of FHL1 as the protein mutated in human reducing body myopathy. J. Clin. Invest. 118:904-912.

30. Carcamo, J., Zentella, A., and Massague, J. 1995. Disruption of transforming growth factor signaling by a mutation that prevents transphosphorylation within the receptor complex. Mol. Cell Biol. 15:1573-1581.

31. Kurisaki, K., et al. 2003. Nuclear factor YY1 inhibits transforming growth factor $\beta$ - and bone morphogenetic protein-induced cell differentiation. Mol. Cell Biol. 23:4494-4510.

32. Somasundaram, K., et al. 1997. Arrest of the cell cycle by the tumour-suppressor BRCA1 requires the CDK-inhibitor p21WAF1/CiP1. Nature. 389:187-190.

33. Ye, Q., Cinar, B., Edlund, M., Chung, L.W., and Zhau, H.E. 2001. Inhibition of growth and cell cycle arrest of ARCaP human prostate cancer cells by ectopic expression of ER- $\alpha$. Mol. Cell. Biochem. 228: $105-110$

34. Ding, L., et al. 2003. Ligand-independent activation of estrogen receptor $\alpha$ by XBP-1. Nucleic Acids Res. 31:5266-5274.

35. Zhang, H., et al. 2005. Stimulatory cross-talk between NFAT3 and estrogen receptor in breast cancer cells. J. Biol. Chem. 280:43188-43197.

36. Aiyar, S.E., et al. 2004. Attenuation of estrogen receptor $\alpha$-mediated transcription through estrogen-stimulated recruitment of a negative elongation factor. Genes Dev. 18:2134-2146.

37. Bates, G.J., et al. 2005. The DEAD box protein p68: a novel transcriptional coactivator of the p53 tumour suppressor. EMBO J. 24:543-553.

38. Lux, C., et al. 2005. Transition from initiation to promoter proximal pausing requires the CTD of RNA polymerase II. Nucleic Acids Res. 33:5139-5144.

39. Weglarz, L., Molin, I., Orchel, A., Parfiniewicz, B., and Dzierzewicz, Z. 2006. Quantitative analysis of the level of p53 and p21WAF1 mRNA in human colon cancer HT-29 cells treated with inositol hexaphosphate. Acta Biochim. Pol. 53:349-356.

40. Castello, R., et al. 2002. Quantitative real-time reverse transcription-PCR assay for urokinase plasminogen activator, plasminogen activator inhibitor type 1 , and tissue metalloproteinase inhibitor type 1 gene expressions in primary breast cancer. Clin. Chem. 48:1288-1295. 